\title{
Unravelling similarities and differences in the role of circular and linear PVT1 in cancer and human disease
}

\author{
Debora Traversa ${ }^{1,4}$, Giorgia Simonetti $\mathbb{D}^{2,4}$, Doron Tolomeo ${ }^{1}$, Grazia Visci ${ }^{1}$, Gemma Macchia ${ }^{1}$, Martina Ghetti ${ }^{2}$, Giovanni Martinelli ${ }^{2}$, \\ Lasse S. Kristensen ${ }^{3}$ and Clelia Tiziana Storlazzi (iD ${ }^{1 \times}$
}

(c) The Author(s) 2021

The plasmacytoma variant translocation 1 (PVT1) is a long non-coding RNA gene involved in human disease, mainly in cancer onset/ progression. Although widely analysed, its biological roles need to be further clarified. Notably, functional studies on PVT1 are complicated by the occurrence of multiple transcript variants, linear and circular, which generate technical issues in the experimental procedures used to evaluate its impact on human disease. Among the many PVT1 transcripts, the linear PVT1 (IncPVT1) and the circular hsa_circ_0001821 (circPVT1) are frequently reported to perform similar pathologic and pro-tumorigenic functions when overexpressed. The stimulation of cell proliferation, invasion and drug resistance, cell metabolism regulation, and apoptosis inhibition is controlled through multiple targets, including MYC, p21, STAT3, vimentin, cadherins, the PI3K/AKT, HK2, $B C L 2$, and CASP3. However, some of this evidence may originate from an incorrect evaluation of these transcripts as two separate molecules, as they share the IncPVT1 exon-2 sequence. We here summarise IncPVT1/circPVT1 functions by mainly focusing on shared pathways, pointing out the potential bias that may exist when the biological role of each transcript is analysed. These considerations may improve the knowledge about IncPVT1/circPVT1 and their specific targets, which deserve further studies due to their diagnostic, prognostic, and therapeutic potential.

British Journal of Cancer (2022) 126:835-850; https://doi.org/10.1038/s41416-021-01584-7

\section{BACKGROUND}

The one-way DNA-RNA-protein paradigm has been outdated since the discovery of non-coding RNA (ncRNA) genes, which account for two-thirds of the total number of human genes [1]. ncRNAs are involved in critical biological processes. They can regulate gene expression at the transcriptional and posttranscriptional levels and are often deregulated in a variety of human diseases [2]. Thus, they may represent potential keystones for the new targeted therapies of incurable diseases, including cancer.

ncRNAs are classified into two subcategories: small and long ncRNAs (IncRNAs), of less and more than $200 \mathrm{nt}$, respectively [3]. To date, despite the identification of many IncRNAs, most of them still need to be functionally characterised.

Circular RNAs (circRNAs) represent a particular subtype of ncRNAs originating from back-splicing events. Discovered 40 years ago, they initially were considered as splicing by-products with unknown functions [4]. Their roles have recently been reevaluated due to the discovery of thousands of circRNA entities. Some of them are highly abundant, evolutionary conserved, and involved in cellular differentiation and tissue homoeostasis, as well as in the development of multiple diseases $[4,5]$. Notably, the majority of circRNAs originate from genes that show oncogenic effects [6].

Great interest has recently been devoted to the "plasmacytoma variant translocation 1" (PVT1) IncRNA gene, which produces both linear and circular transcripts that have been reported to be overexpressed in several cancer types [7]. Interestingly, positive correlations between PVT1 overexpression and tumour progression are frequently observed [8-10] (see the section "Clinical impact of IncPVT1 and circPVT1").

PVT1 maps at the $8 \mathrm{q} 24$ chromosomal band, reported as a gene desert, harbouring two fragile sites (FRA8C and FRA8D) [11]. It is an exceptionally complex locus, which gives rise to 176 linear splicing variants (according to the Ensembl Genome Browser, https://www. ensembl.org/index.html), 27 of which are also reported at the UCSC Genome Browser (https://genome.ucsc.edu/index.html, Fig. 1), as well as to 29 circular RNAs, as reported in the Circlnteractome (https://circinteractome.nia.nih.gov/) [12], and circBase (http://www. circbase.org/) [13] databases (Table 1). In addition, according to the UCSC Genome Browser, the PVT1 locus harbours five highly conserved microRNAs (miRNAs) (Fig. 1). Some linear transcripts were detected by exon-specific RT-qPCR [14], 5'RACE PCR [8], and IncRNA microarrays [15]; others resulted from transcript predictions

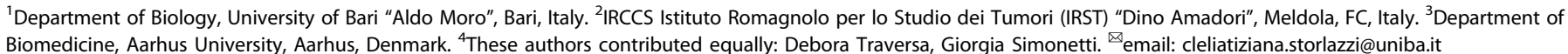

Received: 16 April 2021 Revised: 27 August 2021 Accepted: 4 October 2021

Published online: 9 November 2021 


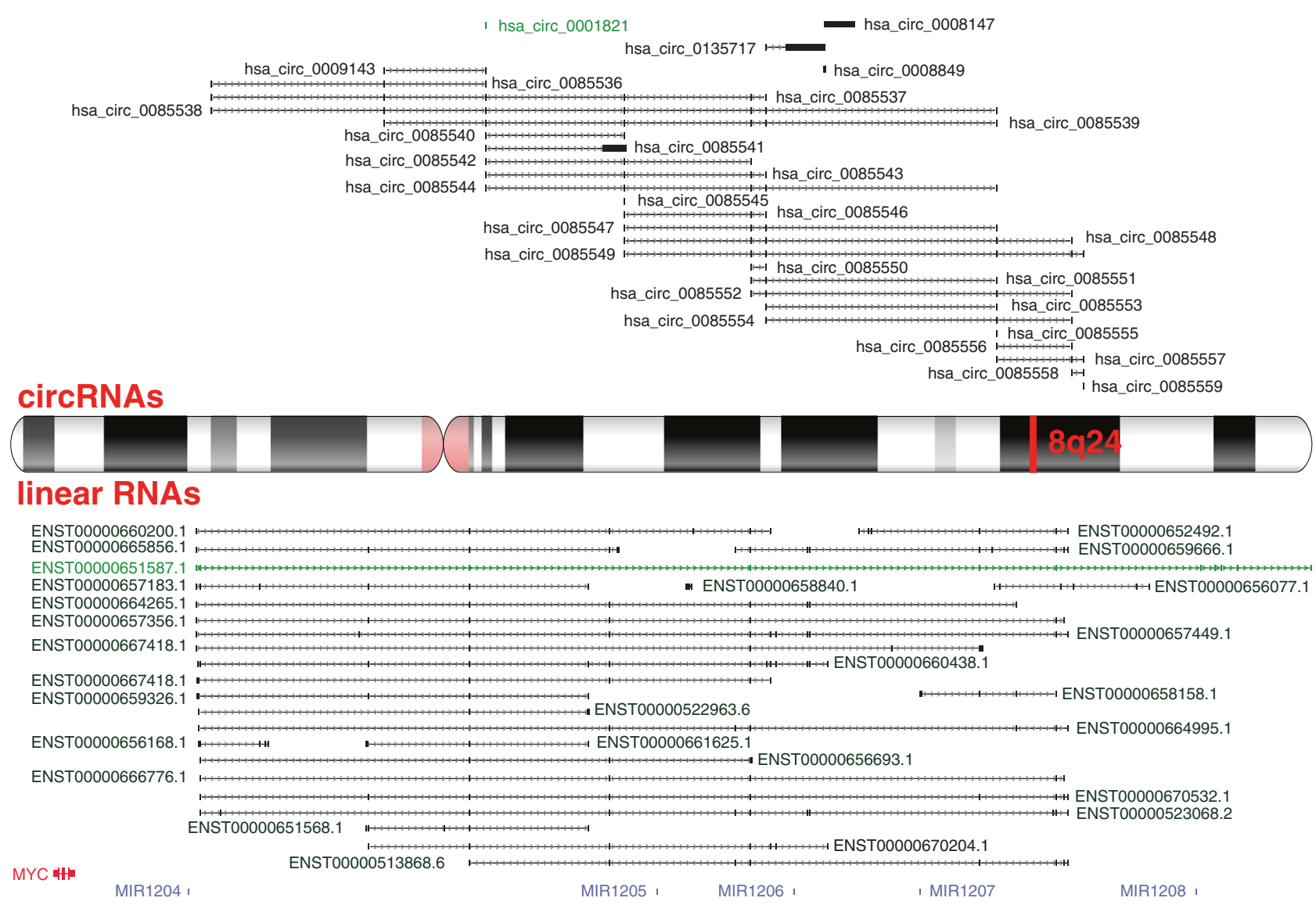

Fig. 1 The PVT1 genomic locus and its circular and linear transcript isoforms. Chromosome 8 ideogram. The circular (from the Circlnteractome and circBase databases) and linear transcripts (from the UCSC Genome Browser) of PVT1 are indicated at the top and bottom parts of the figure, respectively. Each transcript isoform is represented at its correspondent map position on the genome (GRCh38/hg38). The most studied RNA entities are shown in green. MYC (red) and miRNA genes (light purple) are also represented.

by computational approaches. The most extended linear transcript at the PVT1 locus is the PVT1-224/ENST00000651587.1 isoform (herein referred to as IncPVT1). A few reports have addressed the differential roles of such linear and circular transcript variants in cancer and disease so far. For the linear isoforms, the overexpression of different splicing variants was observed in ovarian cancer cell lines [8], gastrointestinal tumours [16], and prostate cancer [14, 17]. For instance, in colorectal cancer (CRC), He et al. identified the overexpression of 14 PVT1 IncRNAs in CRC samples compared with paired adjacent non-tumour tissues using IncRNA microarray [15]. In particular, they focused on the PVT1-214 variant, the most overexpressed one, revealing its role in the upregulation of the Lin28 RNA-binding protein by acting at both transcript (competing with miR-128 for the Lin28 mRNA binding) and protein level. Furthermore, the authors indicated an effect on let-7 miRNA expression, offering a new scenario where the PVT1-214/Lin28/let7 axis serves as a critical regulator of CRC pathogenesis [15]. Moreover, Martínez-Barriocanal et al. reported a role for 11 PVT1 splicing variants as miRNA sponges in gastrointestinal tumours [16] (Table 1).

Other studies pointed at evaluating the expression of the multiple PVT1 transcripts by quantifying exons $4 \mathrm{~A}, 4 \mathrm{~B}$ and 9 in prostate cancer patients [14], even though they did not refer to specific transcript variants. Interestingly, a splicing variant named PVT1b, including exon $1 \mathrm{~b}$ in place of $1 \mathrm{a}$, was described as having tumour suppressor properties [18, 19] (see the section "IncPVT1 upregulation in human disease and cancer"). According to the Ensembl Genome Browser, multiple transcripts include PVT1 exon $1 \mathrm{~b}$.
Conversely, circular PVT1 variants were detected only by bioinformatics tools [20-23] (Table 1). Functional data are limited to the hsa_circ_0001821 circular RNA (herein referred to as circPVT1), which shares the exon-2 full-length sequence (410 nt) with IncPVT1, and the hsa_circ_0009143 [24]. The latter is overexpressed in cervical cancer and is involved in epithelial-mesenchymal transition (EMT), in which normal polarised epithelial cells transform their phenotype and acquire mesenchymal characteristics and metastasis [24]. circPVT1 derives from a back-splicing event, prompted by a loop structure generated by the presence of Alu repeats flanking exon 2 of PVT1 [21]. The circular structure makes circPVT1 resistant to exonuclease cleavage, and therefore, highly stable. Indeed, its half-life exceeds $24 \mathrm{~h}$, while IncPVT1 shows a half-life of fewer than $4 \mathrm{~h}$ [25].

Although InCPVT1 and circPVT1 are different entities, they are often reported in the literature as involved in the same cellular pathways. This review will introduce their specificities and then focus on their shared pathways, downstream molecular targets and the technical issues encountered to study them as separate entities.

\section{THE PVT1 LOCUS IS FREQUENTLY AMPLIFIED AND REARRANGED IN HUMAN CANCER}

Multiple PVT1 genetic variants are described as associated with cancer susceptibility [26-28]. Previous studies mostly documented its involvement in genomic aberrations, e.g., translocations and high copy number amplification, in different malignancies.

Translocations affecting the 8q24 locus are well-documented in multiple myeloma [29], lymphoma [30] and chronic lymphocytic 
II

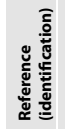

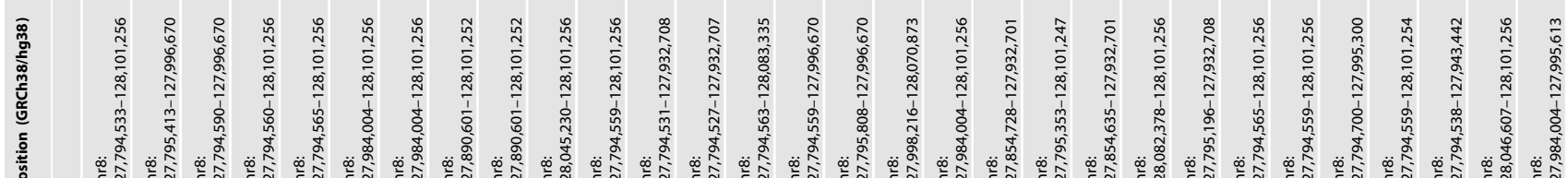

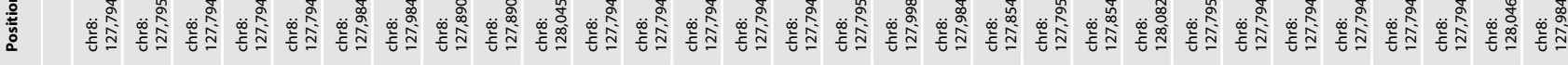
高 总

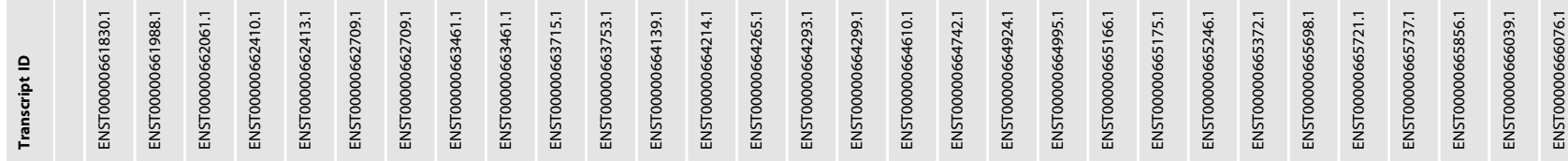

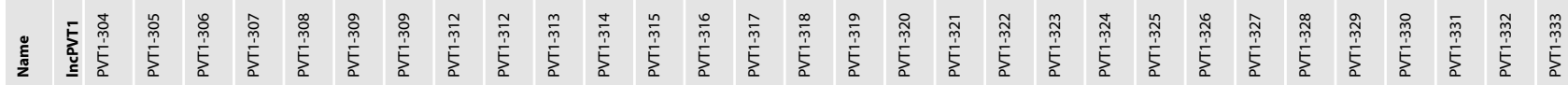
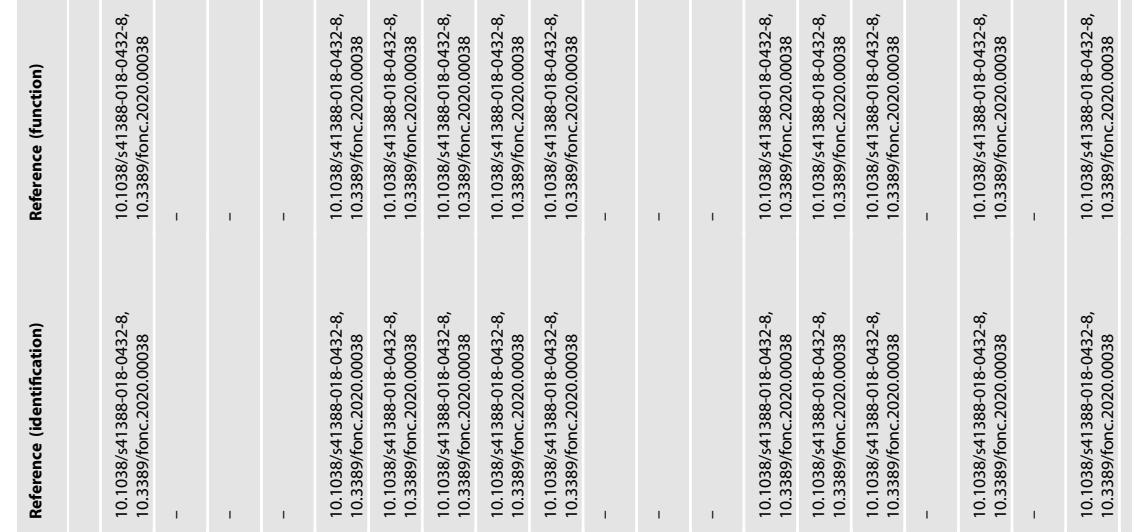

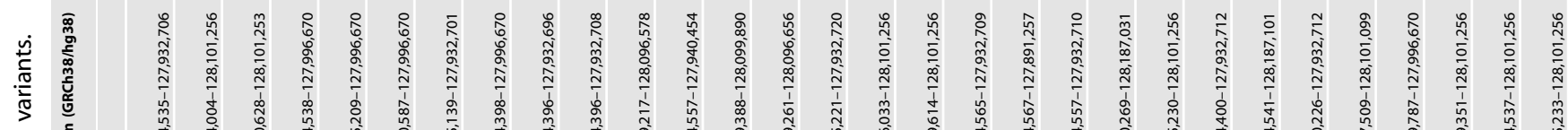

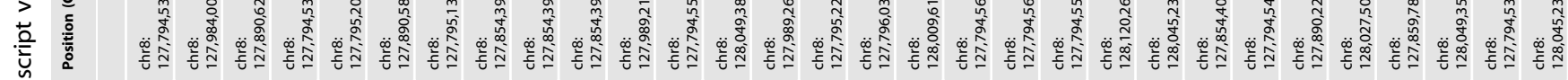

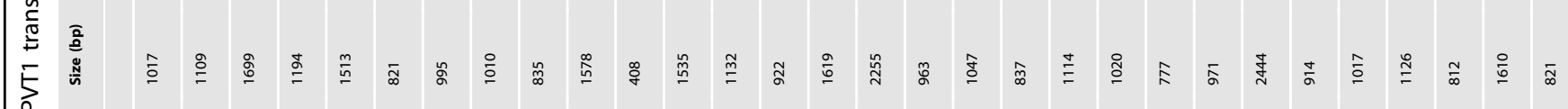
.⿳亠二口犬

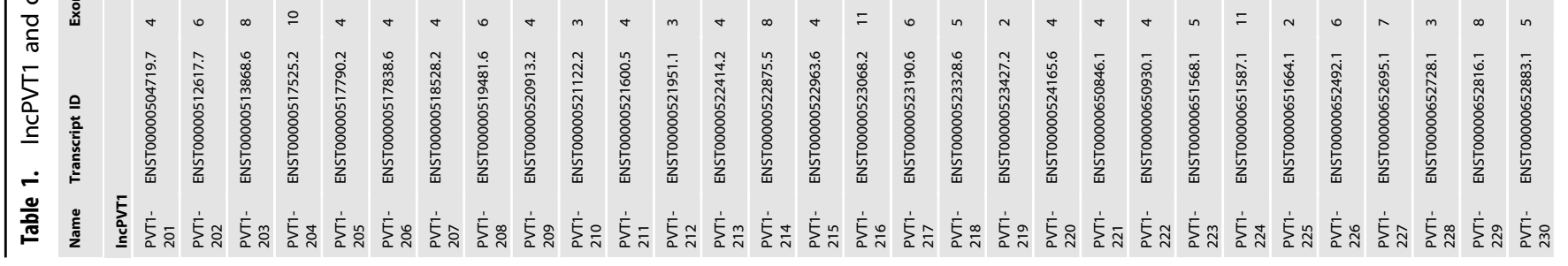




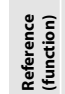

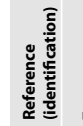

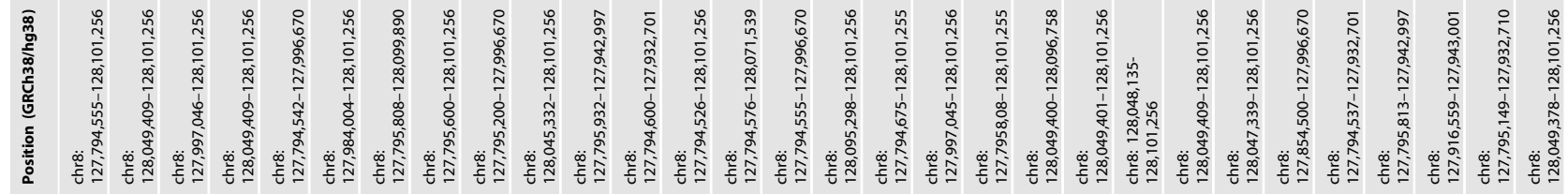

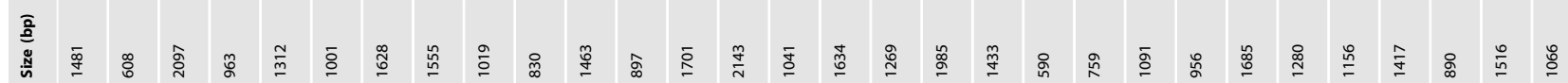

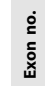

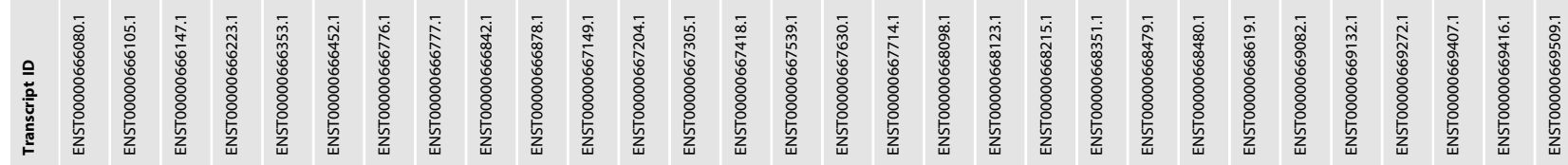

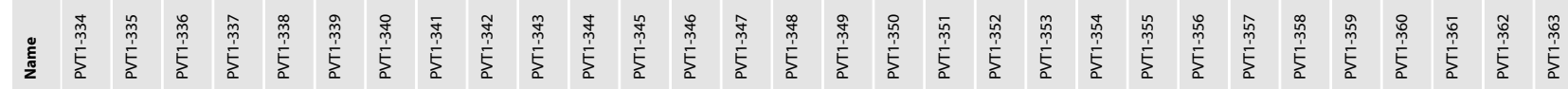

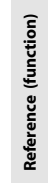

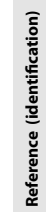

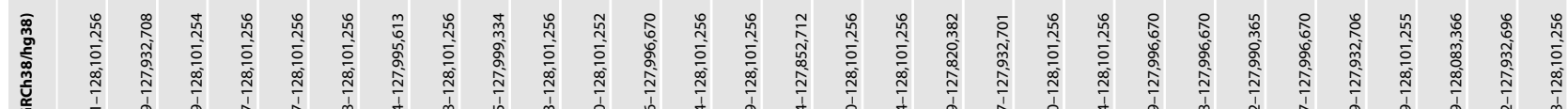

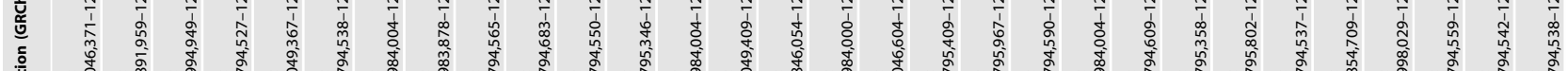

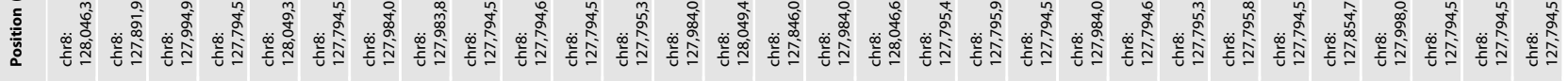
产咅

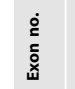

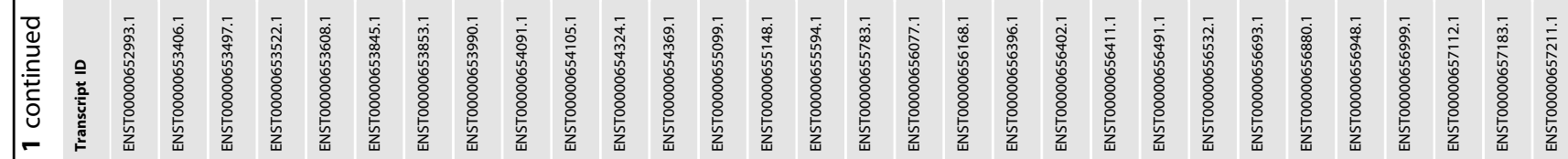

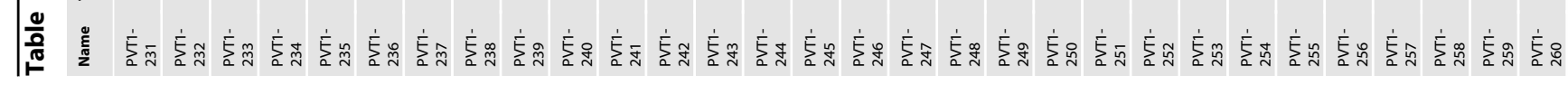


产高

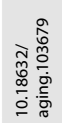
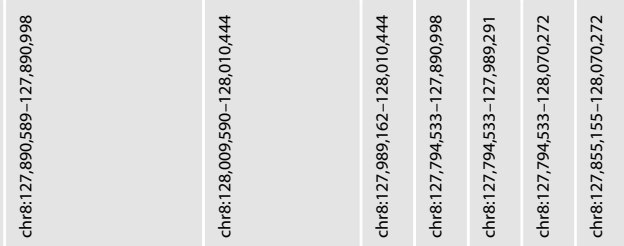

กั้

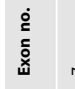

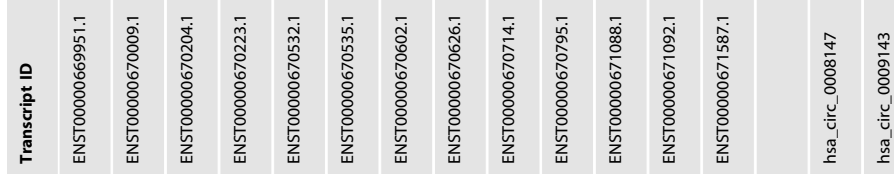

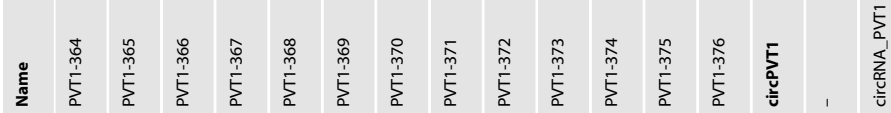

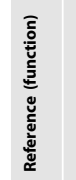

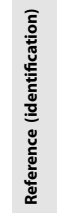

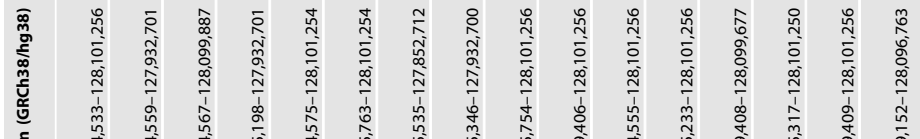

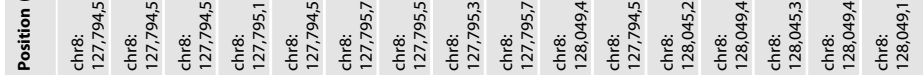

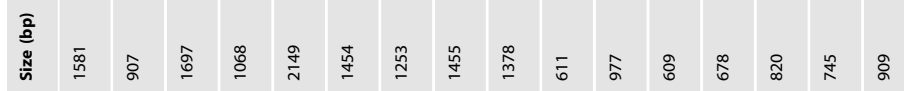

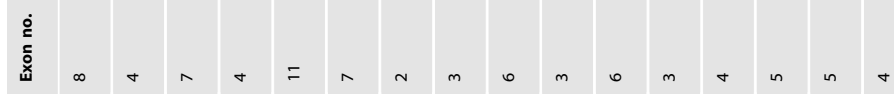

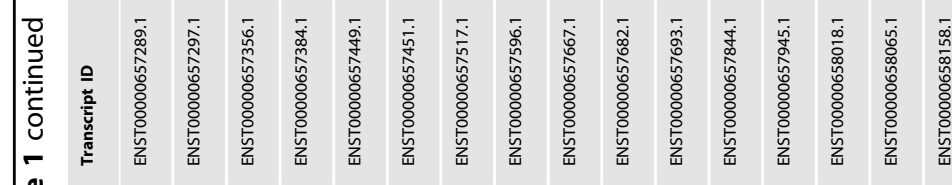

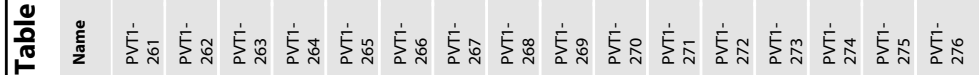

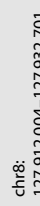

$\tilde{\dddot{\gamma}}$

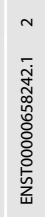

홍쵸

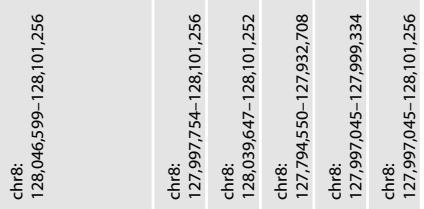

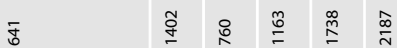

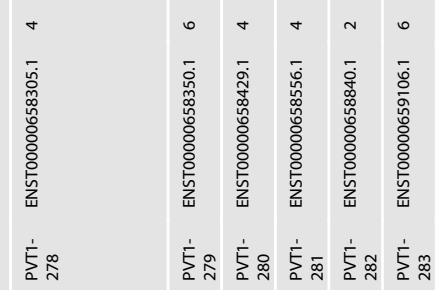




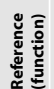

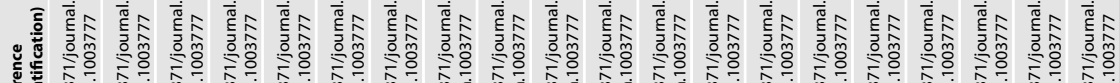

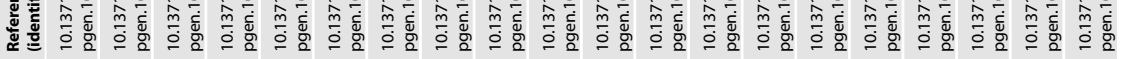

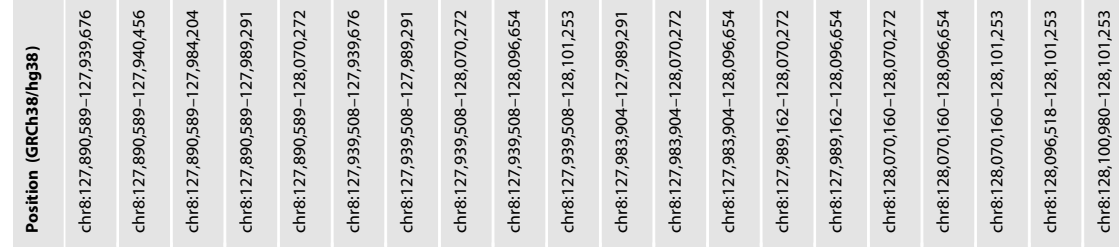
㫄

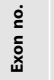

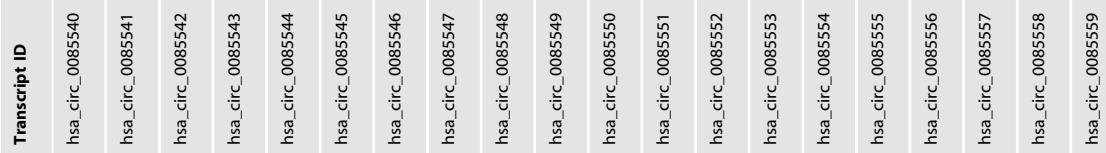

$\stackrel{\frac{0}{2}}{\underline{2}}$

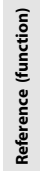

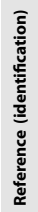

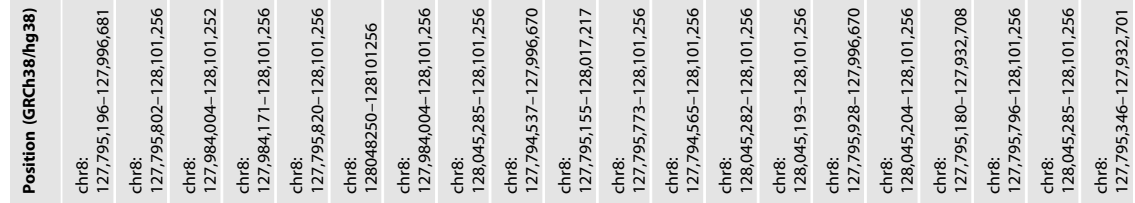
竞

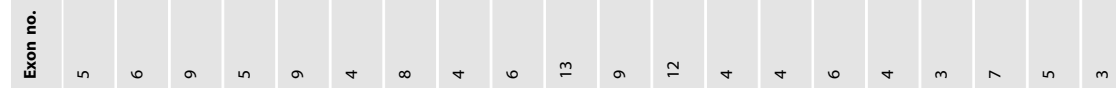

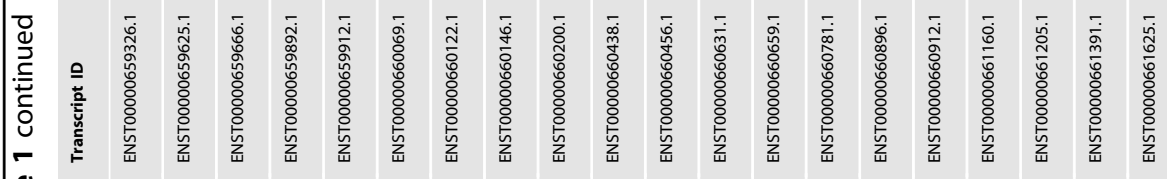

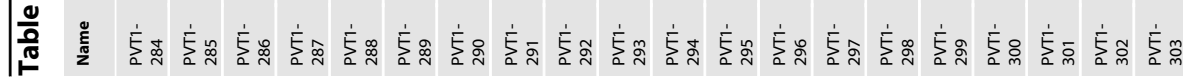


leukaemia [31, 32], and generally result in MYC (located $53 \mathrm{~Kb}$ upstream of PVT1 (Fig. 1)) and PVT1 overexpression; these events are associated with poor prognosis.

Moreover, IncPVT1 has also been reported to be part of fusion transcripts either due to a genomic rearrangement or through trans-splicing events $[33,34]$. However, the potential oncogenic roles of these chimeras have not been investigated yet.

$8 q 24$ high copy number amplification, in the form of double minute chromosomes or homogeneously staining regions, is described in a series of cancers, from haematological malignancies, such as acute myeloid leukaemia [33, 35] and lymphoma [36], to solid tumours, including gastric cancer (GC) [37], small-cell lung cancer (SCLC) [38], breast cancer [39], medulloblastoma [40], ovarian and endometrial cancers [8, 41, 42], and CRC [43].

The $8 q 24$ genomic amplifications usually cause an increased expression of the embedded oncogenes, particularly $M Y C$, even though some exceptions to the amplification-overexpression paradigm are observed [35, 44]. Interestingly, Takahashi et al. demonstrated a stronger correlation between 8q24 copy number gain and PVT1 expression than the one reported between the genomic amplification and MYC [45]. Indeed, a significant amplification of PVT1 alone was found in some tumour types, suggesting that increased PVT1 expression may be sufficient to increase MYC levels, which is crucial in tumorigenesis [46, 47]. Increasing literature documented interactions between MYC and PVT1 at both genomic and transcriptional levels, as also discussed in the section "Cell proliferation". Recent evidence highlighted, in some cancer models (e.g., breast cancer), the role of the PVT1 promoter in the transcriptional regulation of MYC. In detail, Cho et al. identified four PVT1-intergenic enhancers increasing MYC expression when the PVT1 promoter is inactive. The latter acts as a DNA boundary element, modulating enhancer-promoter interactions and displaying a tumour-suppressive role [48]. Although the regulatory action of the PVT1 promoter seems to overcome that of its RNA products, both these elements could contribute to modulating MYC protein levels in a tissue-specific manner. Future studies are needed to clarify the interplay between PVT1-mediated transcriptional and post-transcriptional regulation of MYC.

\section{IncPVT1 upregulation in human disease and cancer}

Independently from genomic events, IncPVT1 is upregulated in tumours relative to normal cells in various cancer types, thus representing a good candidate for targeted therapies [49-51].

Interestingly, You et al. reported the hypomethylation of the PVT1 promoter in several cancer types compared with normal counterparts, suggesting epigenetics as a significant mechanism behind IncPVT1 upregulation [52].

In addition to multiple cellular functions shared between IncPVT1 and circPVT1, which will be discussed later in the review (see the section "Two molecules, same function?"), IncPVT1 is also known to promote angiogenesis, likely by enhancing the expression and secretion of vascular endothelial growth factor (VEGF) [53], and regulating the $W n t / \beta$-catenin axis. IncPVT1 is associated with high cytoplasmic and nuclear $\beta$-catenin levels and expression of its CyclinD1 target [54-56]. The upregulation of the Wnt/ $\beta$-catenin pathway leads to dysregulation of numerous cellular processes, such as cell viability, adhesion, migration, and invasion [57]. Several studies investigated the relationship between PVT1 and Wnt/ $\beta$-catenin, all of which focused on the linear isoform [58-60].

Notably, p53 positively regulates the expression level of the PVT1b isoform through its binding to a p53-responsive element, located about $1200 \mathrm{bp}$ downstream the PVT1 transcriptional start site, between exon $1 \mathrm{a}$ and exon $1 \mathrm{~b}$, also conserved in mice [18]. The activation of this isoform is stress-dependent, as it is heavily induced after treatment of mouse embryonic fibroblasts and murine lung adenocarcinoma KPR cells with genotoxic or oncogenic stress, respectively [19]. Interestingly, PVT1b activation is accompanied by the specific downregulation of Myc transcription, indicating its role as a downstream effector of p53 [19].

This evidence is striking because it underlines the dual behaviour of PVT1 in cancer, either as an oncogene or as a tumour suppressor gene.

In addition to cancer, the aberrant expression of IncPVT1 has been reported in other pathological conditions. For example, Zhang et al. described IncPVT1 as a therapeutic target for obesity treatment due to its role in preadipocyte differentiation and adipogenesis. Interestingly, they found a significant upregulation of this linear transcript in mature adipocytes compared with preadipocytes, impacting the expression of genes involved in the fatty acid synthesis, transportation and lipogenic transcription [61].

Despite these initial reports, there is still a missing link between the upregulation of IncPVT1 and its causative role in human diseases and cancer development and progression. Moreover, the heterogeneity due to the occurrence of many PVT1 linear isoforms, which hamper specific gene silencing and quantification experiments, represents an issue for investigating the roles of each particular transcript.

\section{circPVT1 upregulation in cancer and innate immunity}

circPVT1 was first described in GC [25], where its expression is upregulated compared with normal gastric tissue. It was subsequently reported as upregulated in several other tumours. Still, its role in carcinogenesis and potential relevance as a diagnostic or prognostic biomarker and as a drug target in cancer remains to be clarified.

Interestingly, circPVT1 expression can be regulated by the interaction between the YAP1 transcriptional cofactor, belonging to the Hippo pathway, and the mutated p53 protein (mut-p53) [62]. YAP1 exerts oncogenic effects by increasing cell proliferation and inhibiting apoptosis. Verduci et al. found a higher expression of circPVT1 in head and neck squamous cell carcinoma patients harbouring TP53 mutations than in controls [63]. Using siRNA against mut-p53, they observed a downregulation of circPVT1 expression by $\sim 60 \% 24 \mathrm{~h}$ after the transfection. Conversely, no effect on IncPVT1 expression was observed. The authors showed that YAP1 increases circPVT1 expression, acting at both transcriptional (by binding circPVT1 promoter and enhancing its activity) and post-transcriptional (by binding and stabilising circPVT1) levels. This effect is enhanced by mut-p53, which can bind YAP1 and reinforce its interaction with circPVT1. This event, in turn, results in an increased proportion of cells in the cell cycle $S$ and G2 phases and elevated cell proliferation [63].

Finally, many circRNAs, including circPVT1, have been associated with the regulation of innate immunity [64]. Indeed, through the formation of imperfect 16-26-bp RNA duplexes, these highly stable molecules may function as inhibitors of the doublestranded RNA (dsRNA)-activated protein kinase (PKR), which is involved in the innate immune response. Upon viral or bacterial infection, circRNAs are degraded by the endonuclease, RNAse $L$, resulting in a release and subsequent activation of PKR through autophosphorylation [64]. In addition, individuals affected by systemic lupus erythematosus showed lower levels of many circRNAs in their peripheral blood mononuclear cells, including circPVT1, compared with healthy donors, potentially resulting in an aberrant PKR activation [64].

\section{Two molecules, same function?}

Despite the frequent upregulation of IncPVT1 and circPVT1 in solid tumours and haematological malignancies, their expression levels are poorly correlated [25]. These PVT1 isoforms are transcribed by different promoters [63], therefore, they have to be considered separate transcriptional entities although possibly interconnected.

IncPVT1 is enriched in the nucleus versus the cytosol $[19,65,66]$, as observed by subcellular fractionation and 
subsequent RT-qPCR [19, 65-68], and RNA fluorescence in situ hybridisation $[69,70]$ in several cancer cell lines. Interestingly, IncPVT1 is described as a chromatin modifier [71]. It has been demonstrated to bind the histone methyltransferase Enhancer of Zeste Homolog-2 (EZH2), a catalytic subunit of polycomb-repressive complex 2 (PRC2), leading to the direct histone methylation of several gene promoters, including the angiopoietin-like 4 (ANGPTL4) in cholangiocarcinoma [70] and trophoblast cells [72], the thyroidstimulating hormone receptor (TSHR) in thyroid carcinoma [73], the forkhead box f1 (FOXF1) in breast cancer [74], the large tumour suppressor kinase 2 (LATS2) in non-small-cell lung cancer (NSCLC) [49], the tumour suppressors p15 and p16 in GC [66], the miR-146a in prostate cancer [75], the miR-200c in melanoma [76], and the miR-200b in cervical cancer [77]. IncPVT1 could also recruit DNMT1 via $\mathrm{EZH} 2$ and promote DNA methylation of the miR-18b-5p promoter in gallbladder cancer (GBC) [68]. In liver cancer, instead, IncPVT1 interferes with the recruitment of EZH2 to the MYC promoter, thus altering the methylation status and, hence, enhancing its expression [68, 78].

Moreover, IncPVT1 may act as a scaffold for the histone acetyltransferase KAT2A, leading to the final HIF-1a stability increase in nasopharyngeal tumours [71].

Conversely, circPVT1 shows a prevalent cytoplasmic localisation $[63,79-81]$. Both IncPVT1 and circPVT1 have been proposed to function as competing endogenous RNAs (ceRNAs) [37, 49, 57, 61]. IncPVT1 functions as a ceRNA by sponging several miRNAs, including miR-186 in GC [82], and miR-186-5p in hepatocellular carcinoma [83]. A similar miRNA-sponging role is described for circPVT1, as for miR-497 in NSCLC [84] and head and neck cancer [63], miR-204-5p in breast cancer [85], miR-125b in NSCLC [79] and GC [25], and miR-145 in CRC [86].

Furthermore, InCPVT1 can directly bind the FOXM1 [87] and MYC [88] proteins to stabilise them post-translationally as well as restrict STAT3 [89] and Lin28 protein degradation by the proteasome machinery [15].

circPVT1 and IncPVT1 are largely thought to be involved in the same cellular processes. The main pathways and targets commonly regulated by circPVT1 and IncPVT1 are summarised in the sections "Cell proliferation", "Oncogenesis and tumour progression", "Apoptosis", "Drug resistance", "Cancer metabolism" and "Clinical impact of IncPVT1 and circPVT1", and Fig. 2.

However, some of these observations may result from technical issues related to difficulties in discerning the two as separate entities in particular experimental analyses, as discussed in the section "Technical issues for PVT1 quantification and experimental knockdown".

\section{Cell proliferation}

IncPVT1 and circPVT1 control cell proliferation by regulating target genes such as MYC and CDKN1A (cyclin-dependent kinase inhibitor 1A).

The potential interaction between MYC and PVT1 genes, although widely discussed in the literature, remains controversial. For instance, it is unclear whether these genes may act synergistically, how they are regulated and if PVT1 linear and/or circular isoforms impact MYC transcription and/or translation. In acute lymphoblastic leukaemia (ALL), IncPVT1 increases MYC protein levels with the resulting driver effects on primary tumours [90]. In GC, both circPVT1 and IncPVT1 were described to increase MYC protein levels. circPVT1 facilitates its translation by sequestering let-7b miRNA, whereas, IncPVT1 directly stabilises MYC [25]. IncPVT1, indeed, blocks the phosphorylation of MYC at threonine 58 and prevents its degradation through the ubiquitin-proteasome pathway $[47,88]$. Therefore, enhanced IncPVT1 level may increase MYC activity in cancer cells by impairing its turnover. In turn, MYC can act as a PVT1 transcriptional activator by binding to two E-box elements located at the PVT1 promoter [9].
Both circPVT1 and IncPVT1 impact the expression of the p21 senescence marker, which is encoded by the CDKN1A transcript. By sponging let-7 miRNAs, circPVT1 decreases the level of CDKN1A in fibroblast cells [91]. In pancreatic cancer cells, the silencing of IncPVT1 significantly increases the expression level of this tumour suppressor gene, influencing proliferation and migration [92]. Moreover, in NSCLC, IncPVT1 promotes cell proliferation by downregulating p21. This effect was demonstrated by using specific siRNA against IncPVT1 [93]. Similarly, in the Raji Burkitt lymphoma cell line, after IncPVT1 silencing, an increased level of p21 was observed, with a subsequent cell cycle block in G0/G1 phases [94].

\section{Oncogenesis and tumour progression}

In glioblastoma multiforme (GBM), the upregulation of circPVT1 activates, through miR-199a-5p downregulation, the PIK3/AKT pathway, which promotes tumour progression [95]. Interestingly, IncPVT1 in CRC acts as a ceRNA for the tumour suppressor miR214-3p, leading to increased PIK3/AKT levels, which may cause cancer development [96]. The same effect was observed in human endometrial carcinoma, where IncPVT1 acts through the PVT1/ miR-195-5p/FGFR1-FGF2 axis, whose main downstream targets are PIK3/AKT [97].

Moreover, in hepatoblastoma, IncPVT1 overexpression is associated with high levels of $\mathrm{p}$-STAT3, thus promoting proliferation and cancer progression [98]. Accordingly, in oral squamous cell carcinoma (OSCC), circPVT1 sponges miR-125b, which targets the STAT3 transcript. Therefore, increased circPVT1 levels cause an accumulation of STAT3, leading to tumour growth [80].

STAT3 has a well-defined role in cancer development, acting in the VEGFA transcriptional activation, promoting angiogenesis. In GC, a positive feedback loop has been demonstrated between STAT3 and the IncPVT1 expression: STAT3 overexpression leads to increased transcription of IncPVT1, which stabilises both STAT3 mRNA and protein in the nucleus. IncPVT1 prevents ubiquitin-proteasomal degradation of phosphorylated STAT3 (p-STAT3), resulting in protein accumulation in the nucleus and activation of the STAT3 signalling pathway [89].

Both IncPVT1 and circPVT1 seem to facilitate cell invasion and metastasis by promoting EMT, losing the adhesion-inhibition capabilities [99]. This phenomenon is mediated by the deregulated expression of key EMT regulators (E-cadherin, $\mathrm{N}$-cadherin and Vimentin), as reported in osteosarcoma, hepatocellular carcinoma, pancreatic cancer, melanoma, oesophageal cancer and cervical cancer [54, 76, 100-103]. Overexpression of IncPVT1 or circPVT1 results in decreased E-cadherin levels (responsible for cell adhesion) and increased expression of $\mathrm{N}$-cadherin and Vimentin (forcing an adhesion-independent phenotype).

\section{Apoptosis}

One of the hallmarks of cancer cells is their capability to escape programmed cell death (apoptosis). Failures in the control of apoptosis may cause tumour initiation, progression and metastasis [104]. Some IncRNAs are negative regulators of apoptosis in tumours [105]. It has been reported that IncPVT1 could inhibit apoptosis in GC tissues through the BCL2 anti-apoptotic factor, having the apoptosis effector CASP3 as a downstream target. When InCPVT1 is upregulated, a simultaneous expression increase of $B C L 2$ [105].

In osteosarcoma cells, IncPVT1 regulates BCL2 through miR-195; when IncPVT1 is upregulated, the miR-195 level decreases and $B C L 2$ transcript increases, resulting in inhibition of apoptosis [106].

circPVT1 also regulates $B C L 2$ via miRNAs. In NSCLC patients, circPVT1 regulates the miR-497/BCL2 axis. Indeed, miR-497 shows a binding site at the $3^{\prime} U T R$ of the BCL2 transcript [84].

Finally, in ALL, high levels of circPVT1 sustain BCL2 protein levels, potentially through miR-125 regulation, thus resulting in 


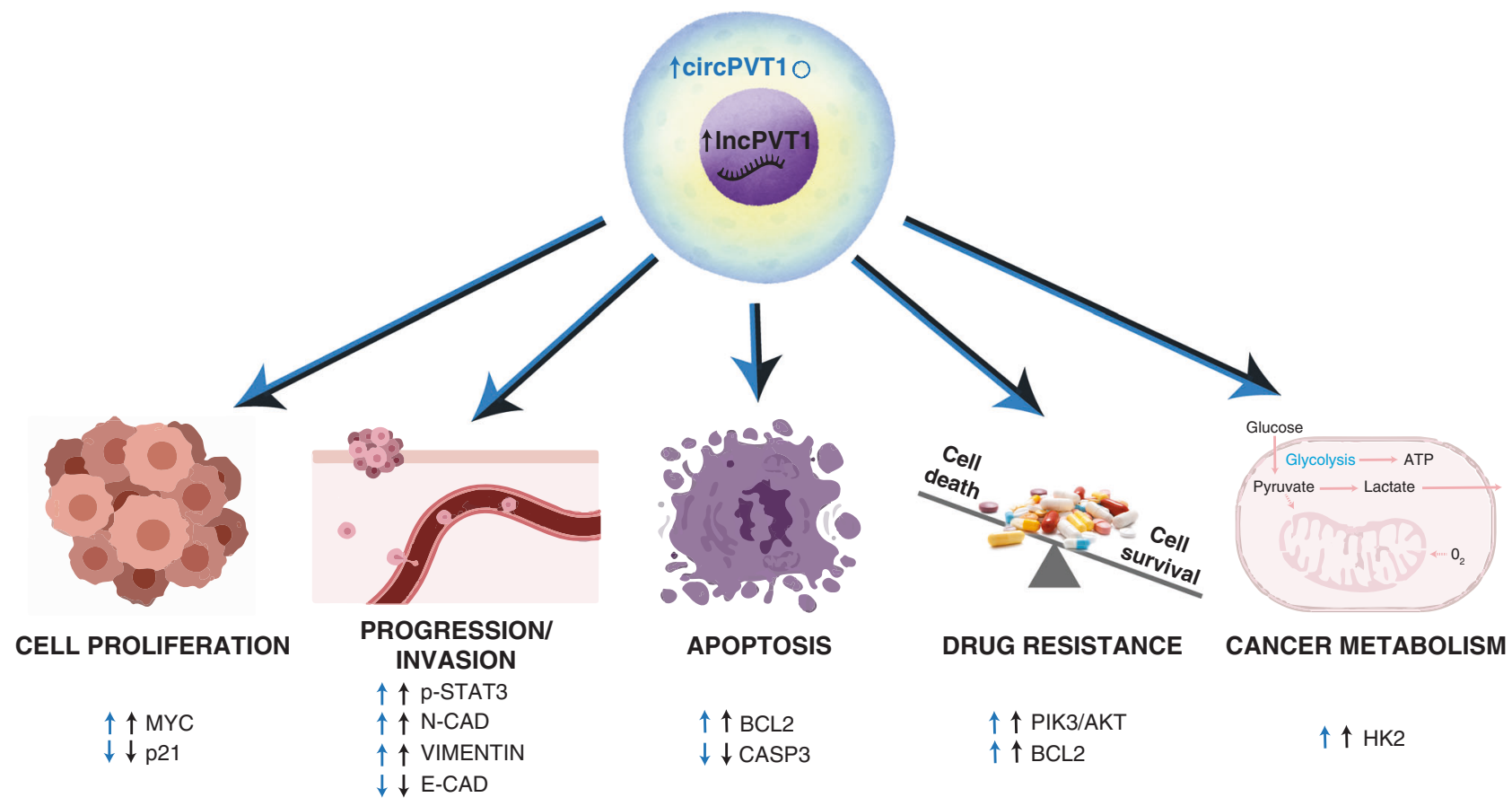

Fig. 2 circPVT1 and IncPVT1 shared features and functions in human cancer cells. Pathways regulated by both circPVT1 and IncPVT1 in cancer, as documented in the literature, are shown. Blue and black arrows indicate functions associated with circPVT1 and IncPVT1, respectively. Upward and downward arrows specify the up- and downregulation of the represented molecules, respectively.

inhibition of apoptosis. circPVT1 may also force BCL2 expression to inhibit MYC-mediated apoptosis [90].

\section{Drug resistance}

Both IncPVT1 and circPVT1 were shown to promote drug resistance in several cancer types by affecting, in some cases, the exact molecular targets. For instance, in osteosarcoma, IncPVT1 modulates miR-152 and prevents its binding to the cmesenchymal-epithelial transition factor (c-MET). This event, in turn, promotes PIK3 activation, inducing drug resistance [107]. The PIK3/AKT pathway is also involved in circPVT1-mediated cisplatin (DDP) resistance in GC. Wang et al. demonstrated that circPVT1 silencing could downregulate the PI3K/AKT signalling through the miR-152-3p/HDGF axis, resulting in decreased DDP resistance and malignancy in GC cells [108].

It has been highlighted that apoptosis and drug resistance are two closely related phenomena in cancer. In GC patients, IncPVT1mediated upregulation of $B C L 2$ seems to enhance drug resistance to the 5-fluorouracil (5-FU), leading to a worse prognosis and shorter overall survival (OS) [105]. Involvement in drug response has also been reported in CRC cells, where IncPVT1 upregulation is associated with 5-FU and DDP resistance. This phenomenon is mediated by $B C L 2$ expression and negative regulation of the apoptotic pathway, influencing BAX and CASP3 pro-apoptotic proteins [109, 110]. In osteosarcoma, circPVT1 upregulation is reported as contributing to doxorubicin (DXR) resistance [111]. Its downregulation in DXR-resistant cell lines resulted in decreased levels of the xenobiotic transmembrane transporters $A B C B 1$ and MRP-1 as well as of BCL2, and increased expression of CASP3 [111].

\section{Cancer metabolism}

Both linear and circular PVT1 can sponge specific miRNAs that modulate HK2 levels. HK2 is involved in cellular metabolism, promoting glucose uptake in cancer cells as a carbon source for aerobic glycolysis [112]. High levels of HK2 are observed in several tumour types and are associated with advanced tumour stage, poor prognosis and metastasis occurrence [113]. A positive correlation between HK2 and IncPVT1 was detected in osteosarcoma, resulting in a high glucose-uptake rate and subsequent lactate production [114]. HK2 is a direct target of miR-497, harbouring a candidate-binding site in its 3'UTR. IncPVT1 promotes tumour development by binding miR-497 and blocking its anticancer effects. Moreover, in GBC, IncPVT1 overexpression causes an increase of both HK2 mRNA and protein by suppressing the miR-143-mediated inhibitory effect [115]. The PVT1/miR-143/ $H K 2$ axis represents the leading target candidate for therapies to regulate cancer metabolism and block tumour progression in GBC.

In OSCC, circPVT1 controls HK2 levels by sponging miR-106a-5p, contributing to cell growth, metastasis and glycolytic metabolism. Interestingly, the 3'-UTR of HK2 mRNA displays a binding site for miR-106a-5p that can directly suppress the protein translation. When circPVT1 is upregulated, miR-106a-5p activity is inhibited, leading to increased expression of $H K 2$, which promotes cancer development [116].

\section{Clinical impact of IncPVT1 and circPVT1}

The altered expression of IncPVT1 and/or circPVT1 has been associated with tumour progression and poor prognosis in several cancer types (Table 2).

In particular, elevated expression levels of IncPVT1 predict poor prognosis and worse clinicopathological characteristics in both solid and haematological malignancies, resulting in a decrease of OS, progression-free survival (PFS) and/or disease-free survival (DFS). Indeed, as reported in Table 2, the IncPVT1 upregulation in tumour tissues is associated with an advanced clinical stage and the presence of lymph node and distant metastases. Similar results were obtained when analysing the clinical impact of circPVT1 overexpression in different solid tumours: it predicted a poor OS and was related with an advanced clinical stage and, when analysed, with the occurrence of lymph node and distant metastases (Table 2). The only exception is GC. In this malignancy, high circPVT1 expression was associated with a good prognosis, likely due to its positive correlation with the tumour suppressor miR-125, which blocks the cell cycle at the G0/G1 phase, seeming to promote apoptosis, and inhibits tumour growth and invasion [25]. 


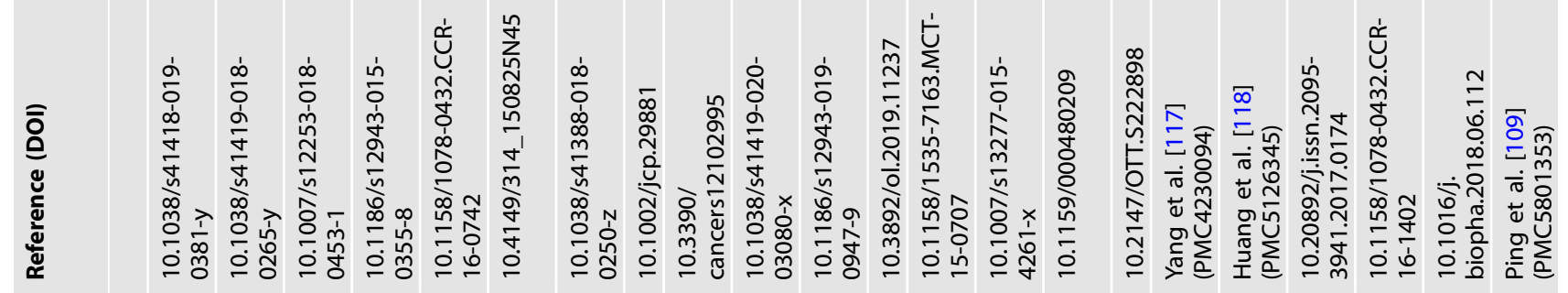

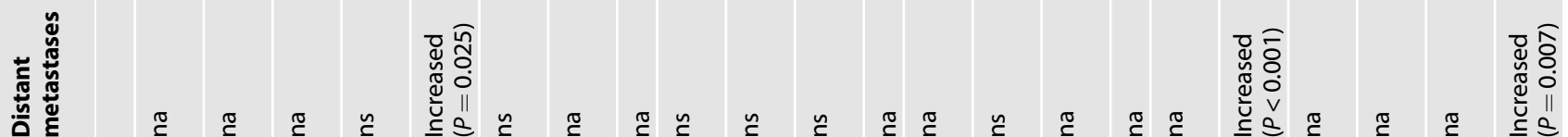

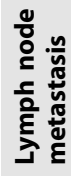

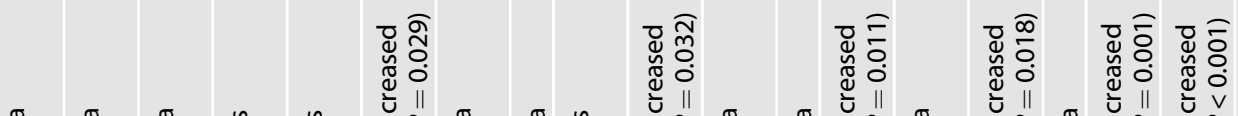

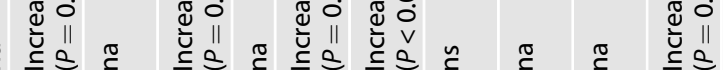

ปัँ

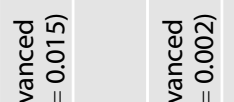

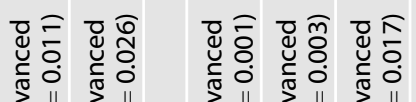

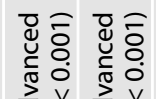

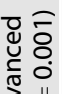

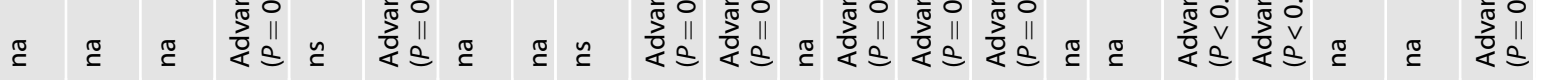

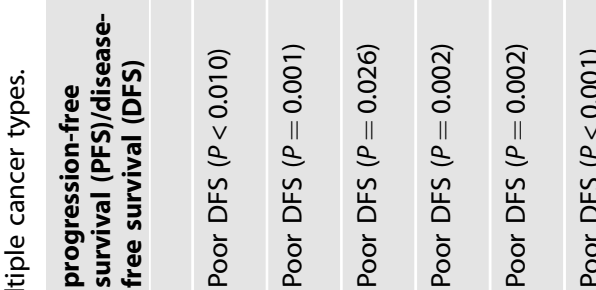

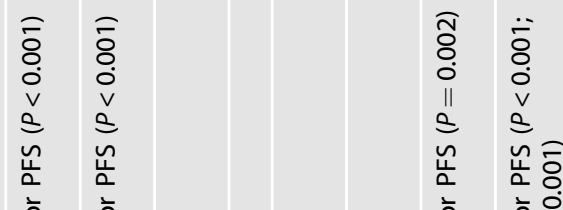

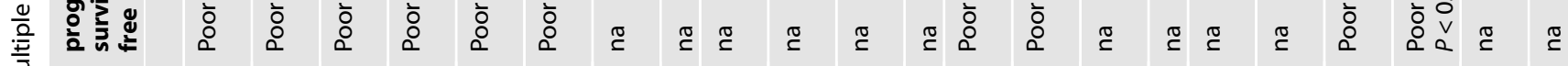

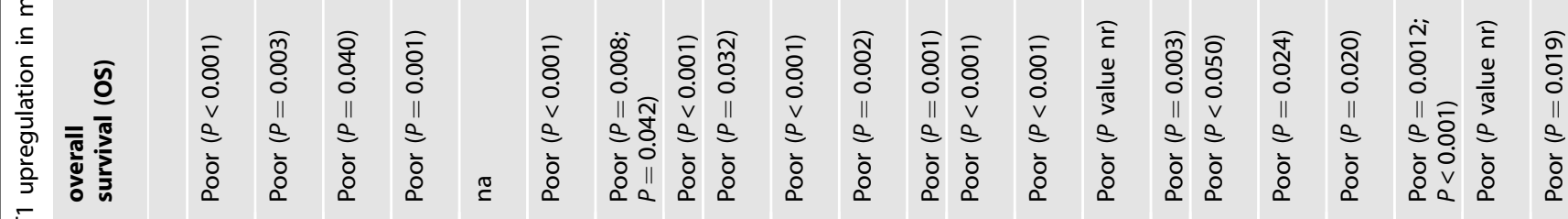

巨

는

至

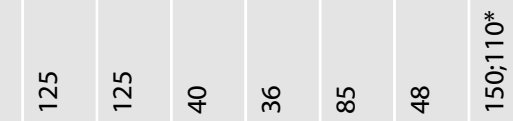

ํํㅇ

동

官

ᄋ ๙

믐

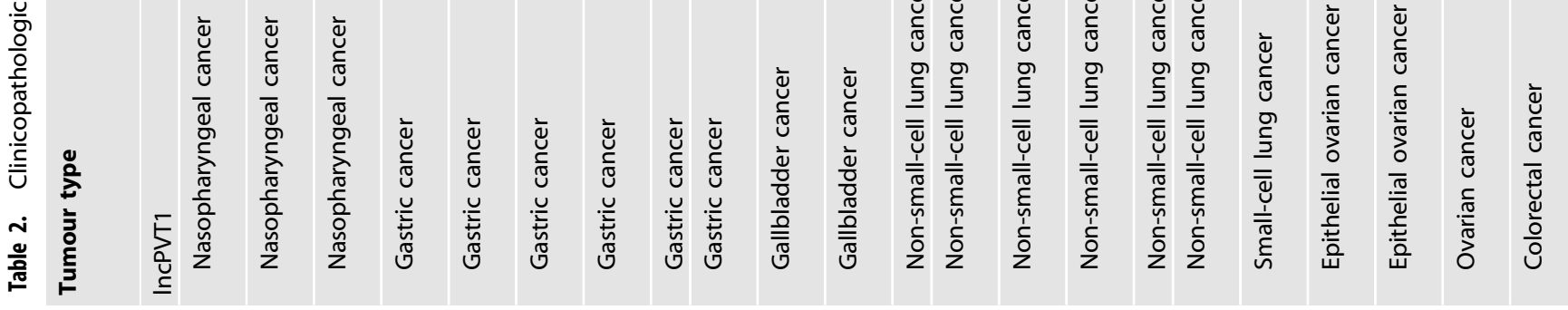

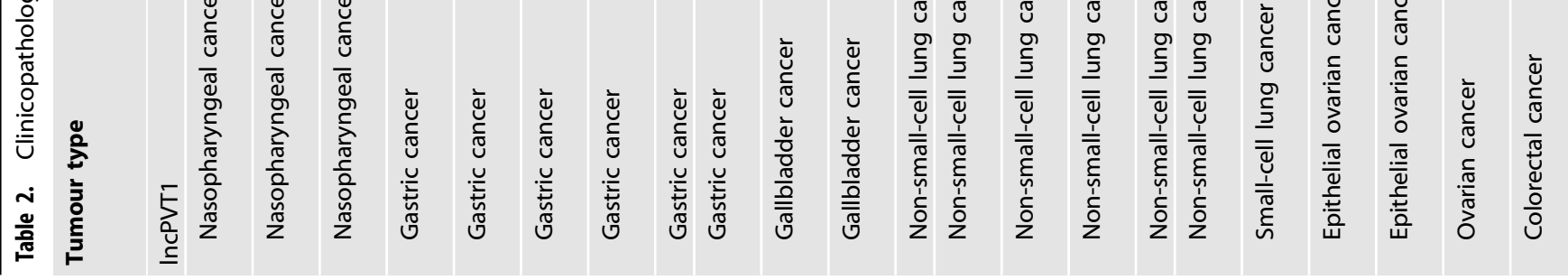




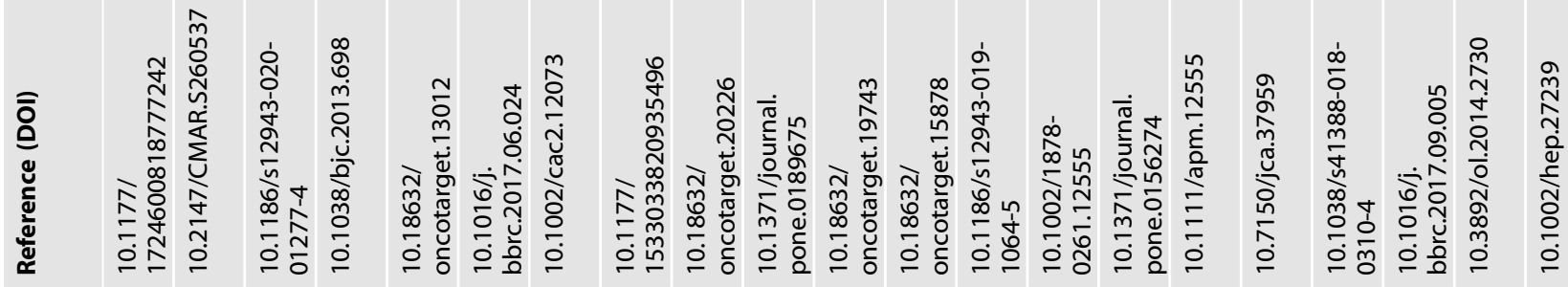

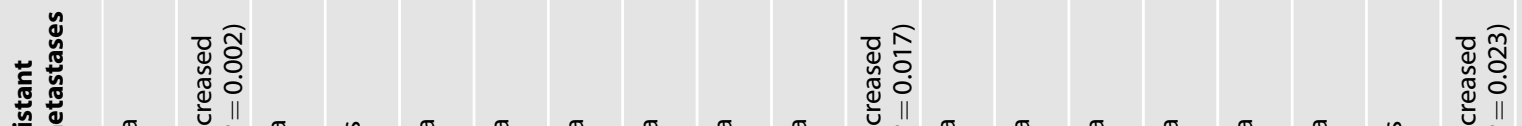

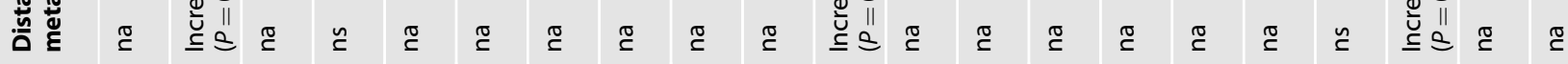

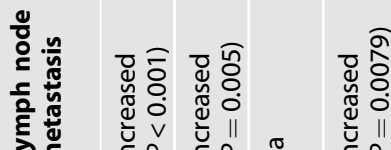

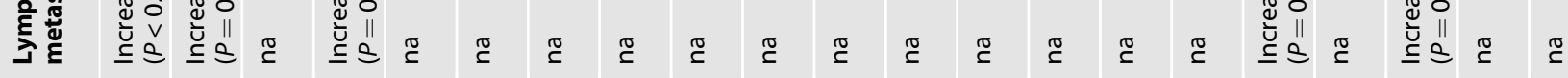

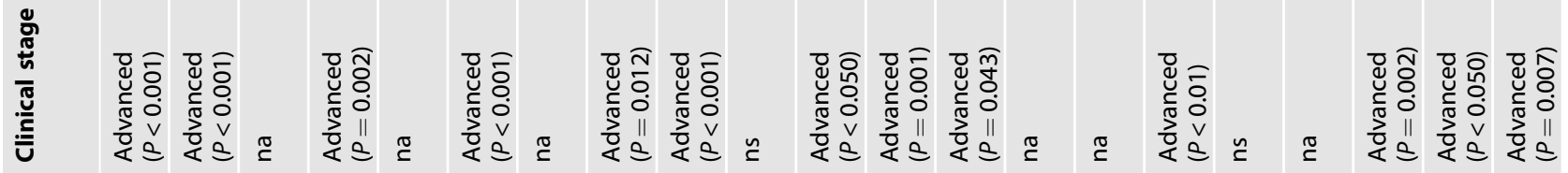

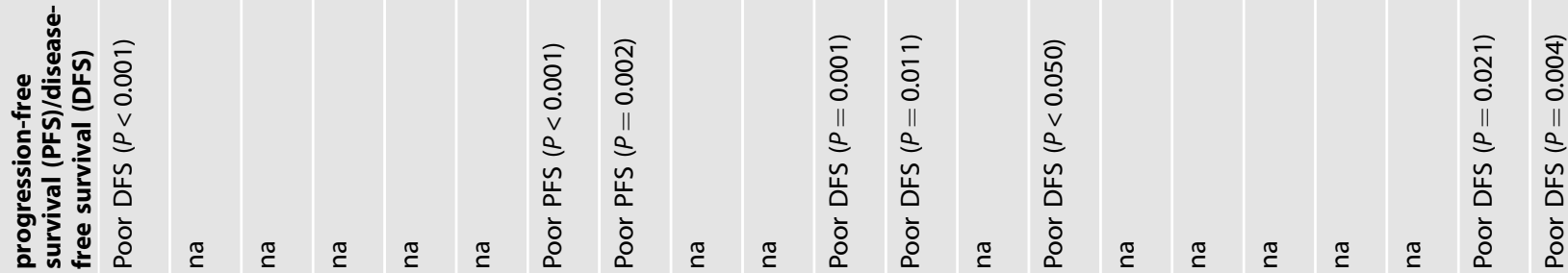

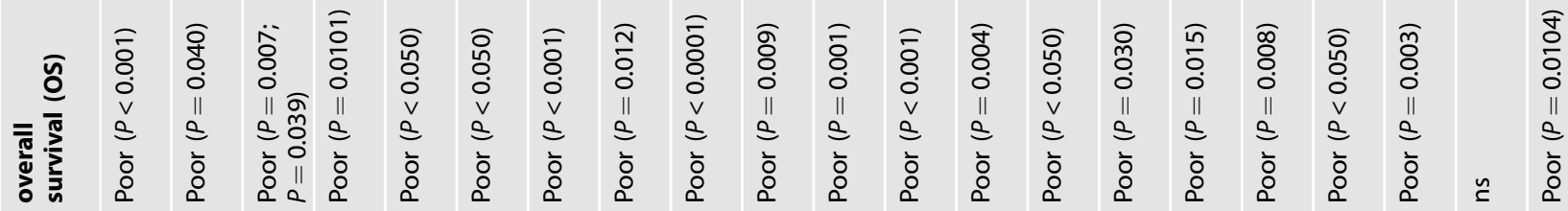

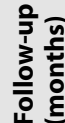

苟亭

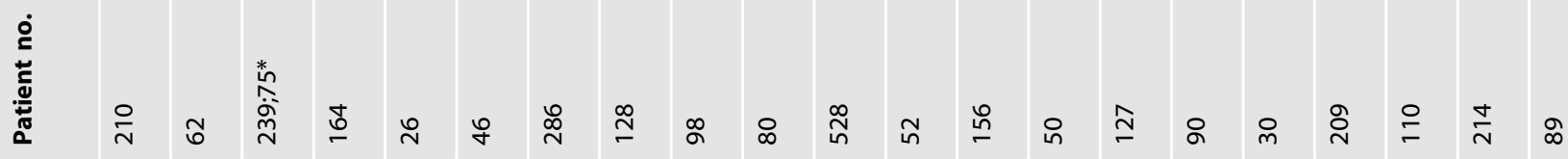

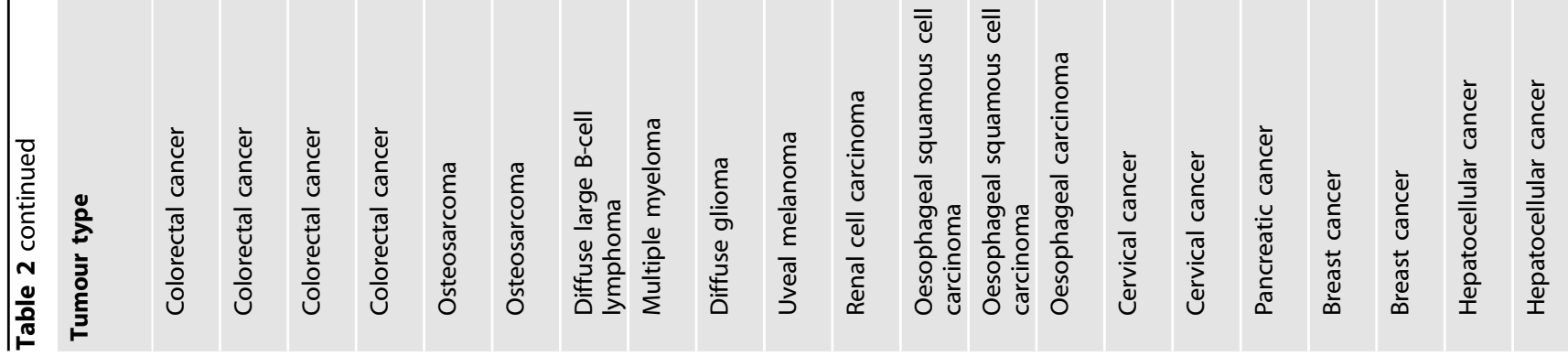




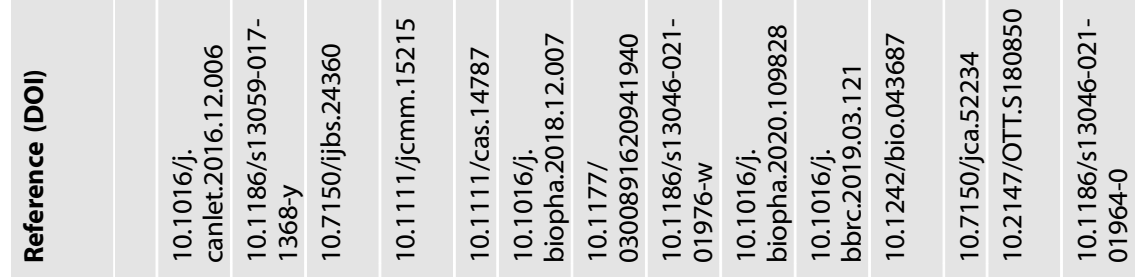

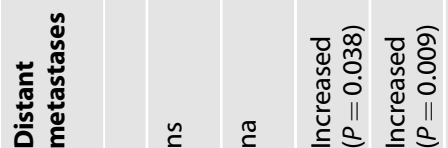

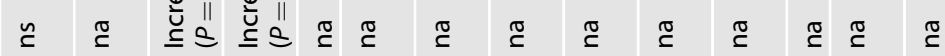

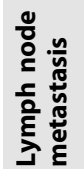

ర্]

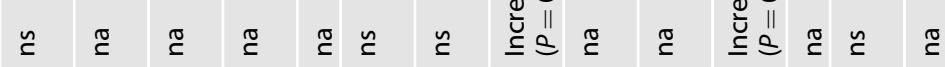

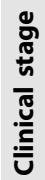

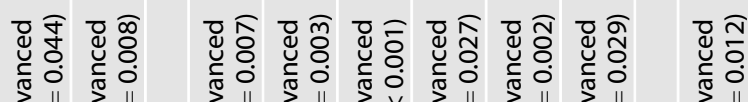

$\leftrightharpoons$ I.

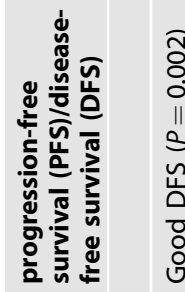

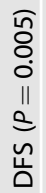

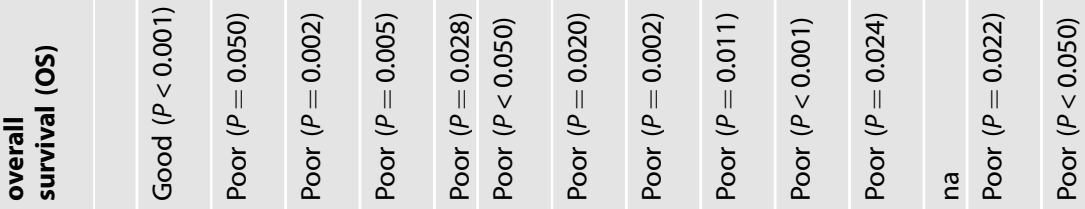

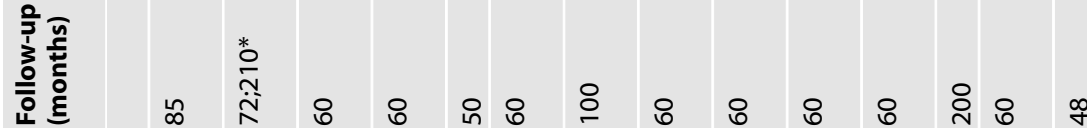

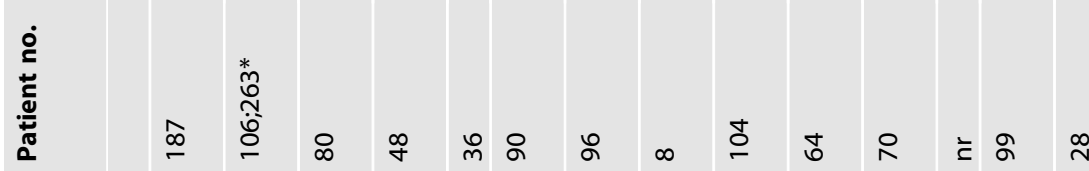

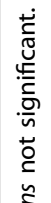

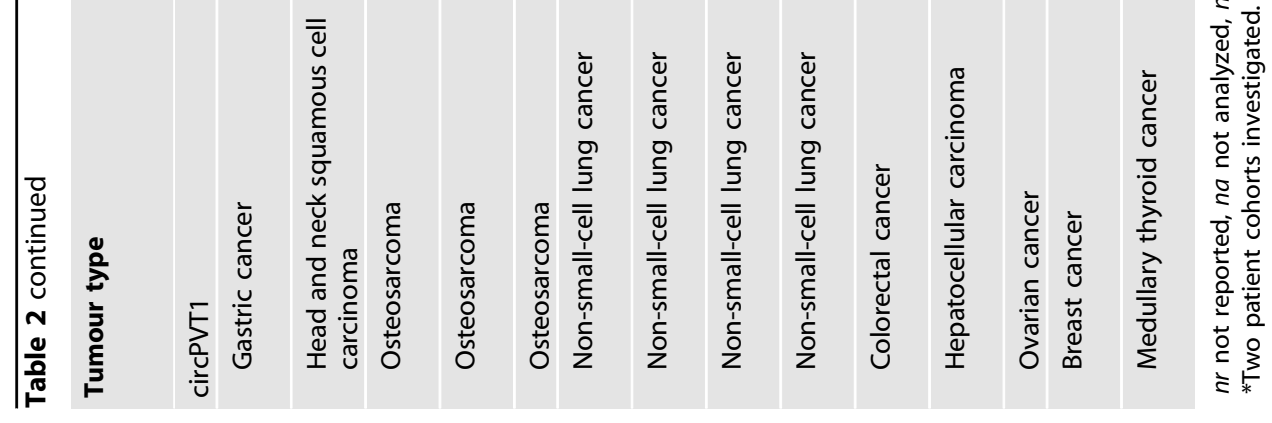




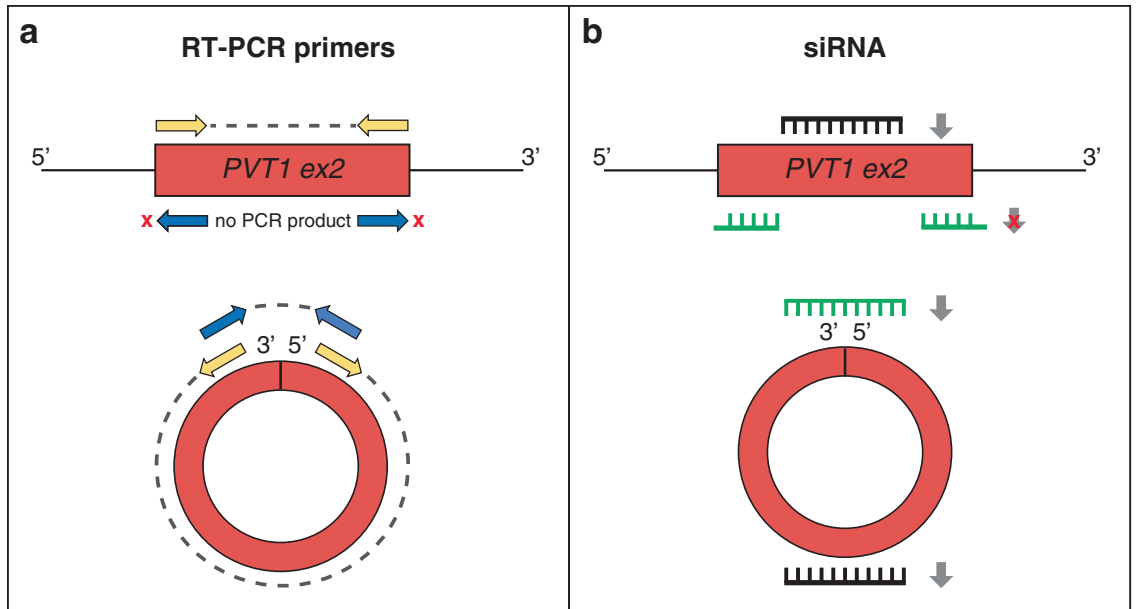

Fig. 3 Technical challenges in PVT1 transcript quantification and silencing assays. a Convergent primers (yellow arrows) designed on IncPVT1 exon 2 give rise to PCR products from both linear and circular isoforms (grey dashed line), whereas divergent primers (blue arrows) amplify a specific PCR product from circPVT1. b A siRNA designed on IncPVT1 exon 2 (black line) silences both IncPVT1 and circPVT1. The specific silencing of circPVT1 can be achieved using a siRNA targeting its back-splicing junction (green line). Grey arrows $=$ transcript silencing.

In summary, both IncPVT1 and circPVT1 might serve as effective prognostic biomarkers for multiple tumour entities.

\section{Technical issues for PVT1 quantification and experimental knockdown}

As IncPVT1 and circPVT1 share the same genomic sequence corresponding to IncPVT1 exon 2, technical approaches capable of discriminating between them are required to understand their individual biological roles.

In this context, we analysed the sequences of primers and siRNAs used to quantify and silence either IncPVT1 or circPVT1 across the literature.

Primers for RT-qPCR assays are often designed within exon 2 of PVT1 with a convergent orientation. If not preceded by RNase $R$ digestion of the linear transcripts, this approach introduces a bias in quantification due to the primer pair annealing to both the circular and linear isoforms, as shown in Fig. 3a. Conversely, divergent primers on exon 2 allow the selective amplification of circPVT1, not requiring preventive digestion of the linear isoform and avoiding issues due to a partial efficiency of this step (Fig. 3a).

Similarly, in knockdown experiments, siRNAs specifically designed on PVT1 exon 2 will not allow a specific inhibition of one of the two isoforms, introducing a bias in evaluating the results, as shown in Fig. 3b. Thus, in a significant fraction of the published papers, it is not clear if the knockdown-related effects are attributable to IncPVT1 or circPVT1 or the result of both being simultaneously silenced. This problem can be overcome by placing the siRNA on the circPVT1 back-splicing junction (Fig. 3b) and a linear splicing junction for IncPVT1.

These technical issues question many published studies claiming specific functions for one of the two isoforms. In particular, the results suggest that both isoforms are involved in the same cellular processes. Thus, more studies are needed to clarify whether the observed effects result from a synergistic action of the two PVT1 isoforms or from technical artefacts.

\section{CONCLUSIONS}

The PVT1 gene has been widely investigated for its roles in cancer. However, the discovery of multiple linear and circular isoforms disclosed its multifaceted activity, with several aspects still to be clarified.

IncPVT1 and circPVT1 have to be considered two distinct entities, possibly sharing certain biological functions and having separate roles in cancer.
The molecular mechanisms behind their involvement in cancer initiation and progression have started to be disentangled. Of note, both transcripts might serve as prognostic biomarkers, and their possible connection with MYC highlights their possible role as targets of future therapies. More work is needed to clarify their potential interactions and roles as distinct transcript entities in cancer, mainly due to technical issues on the distinction between linear and circular isoforms in many published studies.

\section{DATA AVAILABILITY}

Not applicable.

\section{REFERENCES}

1. Losko M, Kotlinowski J, Jura J. Long noncoding RNAs in metabolic syndrome related disorders. Mediators Inflam. 2016;2016:5365209.

2. Esteller M. Non-coding RNAs in human disease. Nat Rev Genet. 2011;12:861-74.

3. Uszczynska-Ratajczak B, Lagarde J, Frankish A, Guigó R, Johnson R. Towards a complete map of the human long non-coding RNA transcriptome. Nat Rev Gene. 2018;19:535-48.

4. Kristensen LS, Hansen TB, Venø MT, Kjems J. Circular RNAs in cancer: opportunities and challenges in the field. Oncogene. 2018;37:555-65.

5. Kristensen LS, Andersen MS, Stagsted LVW, Ebbesen KK, Hansen TB, Kjems J. The biogenesis, biology and characterization of circular RNAs. Nat Rev Genet. 2019;20:675-91.

6. Van Der Steen N, Lyu Y, Hitzler AK, Becker AC, Seiler J, Diederichs S. The circular rna landscape of non-small cell lung cancer cells. Cancers. 2020;12:1091.

7. Ghetti M, Vannini I, Storlazzi CT, Martinelli G, Simonetti G. Linear and circular PVT1 in hematological malignancies and immune response: two faces of the same coin. Mol Cancer. 2020;19:1-15.

8. Guan Y, Kuo WL, Stilwell JL, Takano H, Lapuk AV, Fridlyand J, et al. Amplification of PVT1 contributes to the pathophysiology of ovarian and breast cancer. Clin Cancer Res. 2007;13:5745-55.

9. Carramusa L, Contino F, Ferro A, Minafra L, Perconti G, Giallongo A, et al. The PVT-1 oncogene is a Myc protein target that is overexpressed in transformed cells. J Cell Physiol. 2007;213:511-8.

10. Liu E, Liu Z, Zhou Y, Mi R, Wang D. Overexpression of long non-coding RNA PVT1 in ovarian cancer cells promotes cisplatin resistance by regulating apoptotic pathways. Int J Clin Exp Med. 2015;8:20565.

11. Huppi K, Pitt JJ, Wahlberg BM, Caplen NJ. The $8 q 24$ gene desert: an oasis of noncoding transcriptional activity. Front Genet. 2012;3:69.

12. Dudekula DB, Panda AC, Grammatikakis I, De S, Abdelmohsen K, Gorospe M. Circinteractome: a web tool for exploring circular RNAs and their interacting proteins and microRNAs. RNA Biol. 2016;13:34-42.

13. Glažar $P$, Papavasileiou $P$, Rajewsky N. CircBase: a database for circular RNAs. RNA. 2014;20:1666-70. 
14. Pal G, Ogunwobi OO. Copy number-based quantification assay for non-invasive detection of PVT1-derived transcripts. PLoS ONE. 2019;14:e0226620.

15. He F, Song Z, Chen H, Chen Z, Yang P, Li W, et al. Long noncoding RNA PVT1-214 promotes proliferation and invasion of colorectal cancer by stabilizing Lin28 and interacting with miR-128. Oncogene. 2019;38:164-79.

16. Martínez-Barriocanal Á, Arango D, Dopeso H. PVT1 long non-coding RNA in gastrointestinal cancer. Front Oncol. 2020;10:38.

17. Pal G, Di L, Orunmuyi A, Oluwabunmi Olapade-Olaopa E, Qiu W, Ogunwobi OO. Population differentiation at the PVT1 gene locus: implications for prostate cancer. G3 Genes, Genomes, Genet. 2020;10:2257-64.

18. Barsotti AM, Beckerman R, Laptenko O, Huppi K, Caplen NJ, Prives C. p53dependent induction of PVT1 and miR-1204. J Biol Chem. 2012;287:2509-19.

19. Olivero CE, Martínez-Terroba E, Zimmer J, Liao C, Tesfaye E, Hooshdaran N, et al. p53 activates the long noncoding RNA Pvt1b to inhibit Myc and suppress tumorigenesis. Mol Cell. 2020;77:761-74.

20. Memczak S, Jens M, Elefsinioti A, Torti F, Krueger J, Rybak A, et al. Circular RNAs are a large class of animal RNAs with regulatory potency. Nature. 2013;495:333-8.

21. Jeck WR, Sorrentino JA, Wang K, Slevin MK, Burd CE, Liu J, et al. Circular RNAs are abundant, conserved, and associated with ALU repeats. RNA. 2013;19:141-57.

22. Rybak-Wolf A, Stottmeister C, Glažar $P$, Jens $M$, Pino N, Giusti $S$, et al. Circular RNAs in the mammalian brain are highly abundant, conserved, and dynamically expressed. Mol Cell. 2014;58:870-85.

23. Salzman J, Chen RE, Olsen MN, Wang PL, Brown PO. Cell-type specific features of circular RNA expression. PLoS Genet. 2013;9:e1003777.

24. Wang $H$, Wei $M$, Kang $Y$, Xing J, Zhao $Y$. Circular RNA circ PVT1 induces epithelial-mesenchymal transition to promote metastasis of cervical cancer. Aging. 2020;12:20139.

25. Chen J, Li Y, Zheng Q, Bao C, He J, Chen B, et al. Circular RNA profile identifies circPVT1 as a proliferative factor and prognostic marker in gastric cancer. Cancer Lett. 2017;388: 208-19.

26. Turnbull C, Ahmed S, Morrison J, Pernet D, Renwick A, Maranian M, et al. Genome-wide association study identifies five new breast cancer susceptibility loci. Nat Genet. 2010;42:504-7.

27. Kastler S, Honold L, Luedeke M, Kuefer R, Möller P, Hoegel J, et al. POU5F1P1, a putative cancer susceptibility gene, is overexpressed in prostatic carcinoma. Prostate. 2010;70:666-74

28. Ahmadiyeh N, Pomerantz MM, Grisanzio C, Herman P, Jia L, Almendro V, et al. 8 q24 prostate, breast, and colon cancer risk loci show tissue-specific long-range interaction with MYC. Proc Natl Acad Sci USA. 2010;107:9742-6.

29. Walker BA, Wardell CP, Brioli A, Boyle E, Kaiser MF, Begum DB, et al. Translocations at 8q24 juxtapose MYC with genes that harbor superenhancers resulting in overexpression and poor prognosis in myeloma patients. Blood Cancer J. 2014;4:e191.

30. Boerma EG, Siebert R, Kluin PM, Baudis M. Translocations involving 8q24 in Burkitt lymphoma and other malignant lymphomas: a historical review of cytogenetics in the light of todays knowledge. Leukemia. 2009;23:225-34.

31. Macchia $G$, Lonoce $A$, Venuto $S$, Macrí $E$, Palumbo $O$, Carella $M$, et al. A rare but recurrent $\mathrm{t}(8 ; 13)(\mathrm{q} 24 ; \mathrm{q} 14)$ translocation in B-cell chronic lymphocytic leukaemia causing MYC up-regulation and concomitant loss of PVT1, miR-15/16 and DLEU7. Br J Haematol. 2016;172:296-9.

32. Li Y, Hu S, Wang SA, Li S, Huh YO, Tang Z, et al. The clinical significance of 8q24/ MYC rearrangement in chronic lymphocytic leukemia. Mod Pathol. 2016;29:444-51.

33. L'Abbate A, Tolomeo D, Cifola I, Severgnini M, Turchiano A, Augello B, et al. MYC-containing amplicons in acute myeloid leukemia: genomic structures, evolution, and transcriptional consequences. Leukemia. 2018;32:2152-66.

34. Tolomeo D, Agostini A, Visci G, Traversa D, Storlazzi CT. PVT1: a long non-coding RNA recurrently involved in neoplasia-associated fusion transcripts. Gene. 2021;779:145497.

35. Storlazzi CT, Fioretos T, Surace C, Lonoce A, Mastrorilli A, Strömbeck B, et al. MYC-containing double minutes in hematologic malignancies: evidence in favor of the episome model and exclusion of MYC as the target gene. Hum Mol Genet. 2006;15:933-42.

36. Pophali PA, Marinelli LM, Ketterling RP, Meyer RG, McPhail ED, Kurtin PJ, et al. High level MYC amplification in B-cell lymphomas: is it a marker of aggressive disease? Blood Cancer J. 2020;10:1-9.

37. Kang JU. Chromosome $8 \mathrm{q}$ as the most frequent target for amplification in early gastric carcinoma. Oncol Lett. 2014;7:1139-43.

38. Brägelmann J, Böhm S, Guthrie MR, Mollaoglu G, Oliver TG, Sos ML. Family matters: how MYC family oncogenes impact small cell lung cancer. Cell Cycle. 2017;16:1489--98.

39. Deming SL, Nass SJ, Dickson RB, Trock BJ. C-myc amplification in breast cancer: a meta-analysis of its occurrence and prognostic relevance. $\mathrm{Br} J$ Cancer. 2000;83:1688--95.
40. Zitterbart K, Filkova H, Tomasikova L, Necesalova E, Zambo I, Kantorova D, et al. Low-level copy number changes of MYC genes have a prognostic impact in medulloblastoma. J Neurooncol. 2011;102:25-33.

41. Abeysinghe HR, Cedrone E, Tyan T, Xu J, Wang N. Amplification of C-MYC as the origin of the homogeneous staining region in ovarian carcinoma detected by micro-FISH. Cancer Genet Cytogenet. 1999;114:136-43.

42. Suehiro Y, Umayahara K, Ogata H, Numa F, Yamashita Y, Oga A, et al. Genetic aberrations detected by comparative genomic hybridization predict outcome in patients with endometrioid carcinoma. Genes Chromosom Cancer. 2000;29:75-82.

43. Al-Kuraya K, Novotny H, Bavi P, Siraj AK, Uddin S, Ezzat A, et al. HER2, TOP2A, CCND1, EGFR and C-MYC oncogene amplification in colorectal cancer. J Clin Pathol. 2007;60:768-72.

44. Storlazzi $C T$, Fioretos $T$, Paulsson $K$, Strömbeck B, Lassen $C$, Ahlgren $T$, et al. Identification of a commonly amplified $4.3 \mathrm{Mb}$ region with overexpression of C8FW, but not MYC in MYC-containing double minutes in myeloid malignancies. Hum Mol Genet. 2004;13:1479-85.

45. Takahashi Y, Sawada G, Kurashige J, Uchi R, Matsumura T, Ueo H, et al. Amplification of PVT-1 is involved in poor prognosis via apoptosis inhibition in colorectal cancers. Br J Cancer. 2014;110:164-71.

46. Haverty PM, Hon LS, Kaminker JS, Chant J, Zhang Z. High-resolution analysis of copy number alterations and associated expression changes in ovarian tumors. BMC Med Genomics. 2009;2:1-15.

47. Tseng YY, Moriarity BS, Gong W, Akiyama R, Tiwari A, Kawakami H, et al. PVT1 dependence in cancer with MYC copy-number increase. Nature. 2014;512:82-6.

48. Cho SW, Xu J, Sun R, Mumbach MR, Carter AC, Chen YG, et al. Promoter of IncRNA gene PVT1 is a tumor-suppressor DNA boundary element. Cell. 2018;173:1398-412.

49. Wan L, Sun M, Liu GJ, Wei CC, Zhang EB, Kong R, et al. Long noncoding RNA PVT1 promotes non-small cell lung cancer cell proliferation through epigenetically regulating LATS2 expression. Mol Cancer Ther. 2016;15:1082-94.

50. Derderian C, Orunmuyi AT, Oluwabunmi Olapade-Olaopa E, Ogunwobi OO. PVT1 signaling is a mediator of cancer progression. Front Oncol. 2019;9:502.

51. Wang W, Zhou R, Wu Y, Liu Y, Su W, Xiong W, et al. PVT1 promotes cancer progression via microRNAs. Front Oncol. 2019;9:609.

52. You Z, Xu S, Pang D. Long noncoding RNA PVT1 acts as an oncogenic driver in human pan-cancer. J Cell Physiol. 2020;235:7923-32.

53. Mao Z, Xu B, He L, Zhang G. PVT1 promotes angiogenesis by regulating miR$29 \mathrm{c} /$ vascular endothelial growth factor (VEGF) signaling pathway in non-smallcell lung cancer (NSCLC). Med Sci Monit. 2019;25:5418.

54. Zhang X, Feng W, Zhang J, Ge L, Zhang Y, Jiang X, et al. Long non-coding RNA PVT1 promotes epithelial-mesenchymal transition via the TGF-ß/Smad pathway in pancreatic cancer cells. Oncol Rep. 2018;40:1093-102.

55. Liu Z, Zhang H. LncRNA plasmacytoma variant translocation 1 is an oncogene in bladder urothelial carcinoma. Oncotarget. 2017;8:64273.

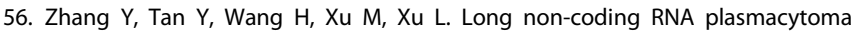
variant translocation 1 (PVT1) enhances proliferation, migration, and epithelialmesenchymal transition (EMT) of pituitary adenoma cells by activating $\beta$-catenin, c-Myc, and cyclin D1 expression. Med Sci Monit. 2019;25:7652.

57. Ghafouri-Fard S, Omrani MD, Taheri M. Long noncoding RNA PVT1: a highly dysregulated gene in malignancy. J Cell Physiol. 2020;235:818-35.

58. Qi G, Li L. Long non-coding RNA PVT1 contributes to cell growth and metastasis in non-small-cell lung cancer by regulating miR-361-3p/SOX9 axis and activating Wnt/ß-catenin signaling pathway. Biomed Pharmacother. 2020;126:110100.

59. Zhou C, Yi C, Yi Y, Qin W, Yan Y, Dong X, et al. LncRNA PVT1 promotes gemcitabine resistance of pancreatic cancer via activating $\mathrm{Wnt} / \beta$-catenin and autophagy pathway through modulating the miR-619-5p/Pygo2 and miR-6195p/ATG14 axes. Mol Cancer. 2020;19:1-24.

60. Yu C, Wang Y, Li G, She L, Zhang D, Chen X, et al. LncRNA PVT1 promotes malignant progression in squamous cell carcinoma of the head and neck. J Cancer. 2018;9:3593.

61. Zhang L, Zhang D, Qin ZY, Li J, Shen ZY. The role and possible mechanism of long noncoding RNA PVT1 in modulating 3T3-L1 preadipocyte proliferation and differentiation. IUBMB Life. 2020;72:1460-7.

62. Di Agostino S, Sorrentino G, Ingallina E, Valenti F, Ferraiuolo M, Bicciato $S$, et al. YAP enhances the pro-proliferative transcriptional activity of mutant p53 proteins. EMBO Rep. 2016;17:188-201.

63. Verduci L, Ferraiuolo M, Sacconi A, Ganci F, Vitale J, Colombo T, et al. The oncogenic role of circPVT1 in head and neck squamous cell carcinoma is mediated through the mutant $\mathrm{p} 53 / \mathrm{YAP} / \mathrm{TEAD}$ transcription-competent complex. Genome Biol. 2017;18:1-24.

64. Liu CX, Li X, Nan F, Jiang S, Gao X, Guo SK, et al. Structure and degradation of circular RNAs regulate PKR activation in innate immunity. Cell. 2019;177:865-80.

65. Cabili MN, Dunagin MC, McClanahan PD, Biaesch A, Padovan-Merhar O, Regev A, et al. Localization and abundance analysis of human IncRNAs at single-cell and single-molecule resolution. Genome Biol. 2015;16:1-16. 
66. Kong R, Zhang EB, Yin DD, You LH, Xu TP, Chen WM, et al. Long noncoding RNA PVT1 indicates a poor prognosis of gastric cancer and promotes cell proliferation through epigenetically regulating $\mathrm{p} 15$ and p16. Mol Cancer. 2015;14:1-14.

67. Videira A, Beckedorff FC, daSilva LF, Verjovski-Almeida S. PVT1 signals an androgen-dependent transcriptional repression program in prostate cancer cells and a set of the repressed genes predicts high-risk tumors. Cell Commun Signal. 2021;19:1-16.

68. Jin L, Cai Q, Wang S, Wang S, Wang J, Quan Z. Long noncoding RNA PVT1 promoted gallbladder cancer proliferation by epigenetically suppressing miR18b-5p via DNA methylation. Cell Death Dis. 2020;11:1-14.

69. Liu DW, Zhang JH, Liu FX, Wang XT, Pan SK, Jiang DK, et al. Silencing of long noncoding RNA PVT1 inhibits podocyte damage and apoptosis in diabetic nephropathy by upregulating FOXA1. Exp Mol Med. 2019;51:1-15.

70. Yu Y, Zhang M, Liu J, Xu B, Yang J, Wang N, et al. Long non-coding RNA PVT1 promotes cell proliferation and migration by silencing ANGPTL4 expression in cholangiocarcinoma. Mol Ther Nucleic Acids. 2018;13:503-13.

71. Wang Y, Chen W, Lian J, Zhang H, Yu B, Zhang M, et al. The IncRNA PVT1 regulates nasopharyngeal carcinoma cell proliferation via activating the KAT2A acetyltransferase and stabilizing HIF-1a. Cell Death Differ. 2020;27:695-710.

72. Xu Y, Lian Y, Zhang Y, Huang S, Zuo Q, Yang N, et al. The long non-coding RNA PVT1 represses ANGPTL4 transcription through binding with EZH2 in trophoblast cell. J Cell Mol Med. 2018;22:1272-82.

73. Zhou Q, Chen J, Feng J, Wang J. Long noncoding RNA PVT1 modulates thyroid cancer cell proliferation by recruiting $\mathrm{EZH} 2$ and regulating thyroid-stimulating hormone receptor (TSHR). Tumor Biol. 2016;37:3105-13.

74. Guo G, Wang F, Han M, Gu Y, Duan X, Li L. Long non-coding RNA PVT1 facilitates cell proliferation by epigenetically regulating FOXF1 in breast cancer. RSC Adv. 2018;8:2740-50.

75. Liu HT, Fang L, Cheng YX, Sun Q. LncRNA PVT1 regulates prostate cancer cell growth by inducing the methylation of miR-146a. Cancer Med. 2016;5:3512-9.

76. Chen L, Ma D, Li Y, Li X, Zhao L, Zhang J, et al. Effect of long non-coding RNA PVT1 on cell proliferation and migration in melanoma. Int J Mol Med. 2018;41:1275-82.

77. Zhang S, Zhang G, Liu J. Long noncoding RNA PVT1 promotes cervical cancer progression through epigenetically silencing miR-200b. APMIS. 2016;124:649-58.

78. Jiang B, Yang B, Wang Q, Zheng X, Guo Y, Lu W. LncRNA PVT1 promotes hepatitis $B$ virus-positive liver cancer progression by disturbing histone methylation on the c-Myc promoter. Oncol Rep. 2020;43:718-26.

79. Li X, Zhang Z, Jiang H, Li Q, Wang R, Pan H, et al. Circular RNA circPVT1 promotes proliferation and invasion through sponging miR-125b and activating E2F2 signaling in non-small cell lung cancer. Cell Physiol Biochem. 2018;51:2324-40.

80. He T, Li X, Xie D, Tian L. Overexpressed circPVT1 in oral squamous cell carcinoma promotes proliferation by serving as a miRNA sponge. Mol Med Rep. 2019:20:3509-18.

81. Bu N, Dong Z, Zhang L, Zhu W, Wei F, Zheng S. Circpvt1 regulates cell proliferation, apoptosis and glycolysis in hepatocellular carcinoma via mir-377/ trim23 axis. Cancer Manag Res. 2020;12:12945.

82. Huang T, Liu HW, Chen JQ, Wang SH, Hao LQ, Liu M, et al. The long noncoding RNA PVT1 functions as a competing endogenous RNA by sponging miR-186 in gastric cancer. Biomed Pharmacother. 2017;88:302-8.

83. Lan T, Yan X, Li Z, Xu X, Mao Q, Ma W, et al. Long non-coding RNA PVT1 serves as a competing endogenous RNA for miR-186-5p to promote the tumorigenesis and metastasis of hepatocellular carcinoma. Tumor Biol. 2017;39:1010428317705338.

84. Qin S, Zhao Y, Lim G, Lin H, Zhang X, Zhang X. Circular RNA PVT1 acts as a competing endogenous RNA for miR-497 in promoting non-small cell lung cancer progression. Biomed Pharmacother. 2019;111:244-50.

85. Bian Q. Circular rna pvt1 promotes the invasion and epithelial-mesenchymal transition of breast cancer cells through serving as a competing endogenous rna for mir-204-5p. Onco Targets Ther. 2019;12:11817.

86. Wang Z, Su M, Xiang B, Zhao K, Qin B. Circular RNA PVT1 promotes metastasis via miR-145 sponging in CRC. Biochem Biophys Res Commun. 2019;512:716-22.

87. Xu MD, Wang Y, Weng W, Wei $P$, Qi P, Zhang Q, et al. A positive feedback loop of IncRNA-PVT1 and FOXM1 facilitates gastric cancer growth and invasion. Clin Cancer Res. 2017;23:2071-80.

88. Yeh E, Cunningham M, Arnold H, Chasse D, Monteith T, Ivaldi G, et al. A signalling pathway controlling c-Myc degradation that impacts oncogenic transformation of human cells. Nat Cell Biol. 2004;6:308-18.

89. Zhao J, Du P, Cui P, Qin Y, Hu C, Wu J, et al. LncRNA PVT1 promotes angiogenesis via activating the STAT3/VEGFA axis in gastric cancer. Oncogene. 2018;37:4094-109.

90. Hu J, Han Q, Gu Y, Ma J, McGrath M, Qiao F, et al. Circular RNA PVT1 expression and its roles in acute lymphoblastic leukemia. Epigenomics. 2018;10:723-32.

91. Panda AC, Grammatikakis I, Kim KM, De S, Martindale JL, Munk R, et al. Identification of senescence-associated circular RNAs (SAC-RNAs) reveals senescence suppressor CircPVT1. Nucleic Acids Res. 2017;45:4021-35.
92. Wu BQ, Jiang $Y$, Zhu F, Sun DL, He XZ. Long noncoding RNA PVT1 promotes EMT and cell proliferation and migration through downregulating p21 in pancreatic cancer cells. Technol Cancer Res Treat. 2017;16:819-27.

93. Cui D, Yu CH, Liu M, Xia QQ, Zhang YF, Jiang WL. Long non-coding RNA PVT1 as a novel biomarker for diagnosis and prognosis of non-small cell lung cancer. Tumor Biol. 2016;37:4127-34.

94. Zheng C, Xiao Y, Li Y, He D. Knockdown of long non-coding RNA PVT1 inhibits the proliferation of raji cells through cell cycle regulation. Oncol Lett. 2019;18:1225-34.

95. Chi G, Yang F, Xu D, Liu W. Silencing hsa_circ_PVT1 (circPVT1) suppresses the growth and metastasis of glioblastoma multiforme cells by up-regulation of miR-199a-5p. Artif Cells, Nanomed Biotechnol. 2020;48:188-96.

96. Shang $A Q$, Wang WW, Yang YB, Gu CZ, Ji $P$, Chen $C$, et al. Knockdown of long noncoding RNA PVT1 suppresses cell proliferation and invasion of colorectal cancer via upregulation of microRNA-214-3p. Am J Physiol Gastrointest Liver Physiol. 2019;317:G222-32.

97. Kong $\mathrm{F}$, Ma J, Yang $\mathrm{H}$, Yang D, Wang $\mathrm{C}$, Ma X. Long non-coding RNA PVT1 promotes malignancy in human endometrial carcinoma cells through negative regulation of miR-195-5p. Biochim Biophys Acta Mol Cell Res. 2018;1865:1479-90.

98. Luo Z, Cao P. Long noncoding RNA PVT1 promotes hepatoblastoma cell proliferation through activating STAT3. Cancer Manag Res. 2019;11:8517.

99. Lamouille S, Xu J, Derynck R. Molecular mechanisms of epithelial-mesenchymal transition. Nat Rev Mol Cell Biol. 2014;15:178-96.

100. Chang QQ, Chen CY, Chen Z, Chang S. LncRNA PVT1 promotes proliferation and invasion through enhancing Smad3 expression by sponging miR-140-5p in cervical cancer. Radiol Oncol. 2019;53:443.

101. Xu Y, Luo X, He W, Chen G, Li Y, Li W, et al. Long non-coding RNA PVT1/miR-150/ HIG2 axis regulates the proliferation, invasion and the balance of iron metabolism of hepatocellular carcinoma. Cell Physiol Biochem. 2018;49:1403-19.

102. Liu YP, Wan J, Long F, Tian J, Zhang C. CircPVT1 facilitates invasion and metastasis by regulating miR-205-5p/c-FLIP axis in osteosarcoma. Cancer Manag Res. 2020;12:1229.

103. Zheng $\mathrm{X}, \mathrm{Hu} \mathrm{H}$, Li S. High expression of IncRNA PVT1 promotes invasion by inducing epithelial-to-mesenchymal transition in esophageal cancer. Oncol Lett. 2016;12:2357-62.

104. Goldar S, Khaniani MS, Derakhshan SM, Baradaran B. Molecular mechanisms of apoptosis and roles in cancer development and treatment. Asian Pacific J Cancer Prevention. 2015;16:2129-44.

105. Du P, Hu C, Qin Y, Zhao J, Patel R, Fu Y, et al. LncRNA PVT1 mediates antiapoptosis and 5-fluorouracil resistance via increasing $\mathrm{Bcl} 2$ expression in gastric cancer. J Oncol. 2019;2019:9325407.

106. Zhou Q, Chen F, Zhao J, Li B, Liang Y, Pan W, et al. Long non-coding RNA PVT1 promotes osteosarcoma development by acting as a molecular sponge to regulate miR-195. Oncotarget. 2016;7:82620.

107. Sun ZY, Jian YK, Zhu HY, Li B. IncRNAPVT1 targets miR-152 to enhance chemoresistance of osteosarcoma to gemcitabine through activating c-MET/PI3K/ AKT pathway. Pathol Res Pract. 2019;215:555-63.

108. Wang X, Zhang Y, Li W, Liu X. Knockdown of cir_RNA PVT1 elevates gastric cancer cisplatin sensitivity via sponging miR-152-3p. J Surg Res. 2021;261:185-95.

109. Ping G, Xiong W, Zhang L, Li Y, Zhang Y, Zhao Y. Silencing long noncoding RNA PVT1 inhibits tumorigenesis and cisplatin resistance of colorectal cancer. Am J Transl Res. 2018;10:138-49.

110. Fan $\mathrm{H}$, Zhu JH, Yao XQ. Knockdown of long non-coding RNA PVT1 reverses multidrug resistance in colorectal cancer cells. Mol Med Rep. 2018;17:8309-15.

111. Kun-Peng Z, Xiao-Long M, Chun-Lin Z. Overexpressed circPVT1, a potential new circular RNA biomarker, contributes to doxorubicin and cisplatin resistance of osteosarcoma cells by regulating ABCB1. Int J Biol Sci. 2018;14:321.

112. Yin C, Qie S, Sang N. Carbon source metabolism and its regulation in cancer cells. Crit Rev Eukaryotic Gene Expression. 2012;22:17-35.

113. Patra KC, Wang Q, Bhaskar PT, Miller L, Wang Z, Wheaton W, et al. Hexokinase 2 is required for tumor initiation and maintenance and its systemic deletion is therapeutic in mouse models of cancer. Cancer Cell. 2013;24:213-28.

114. Song J, Wu X, Liu F, Li M, Sun Y, Wang Y, et al. Long non-coding RNA PVT1 promotes glycolysis and tumor progression by regulating miR-497/HK2 axis in osteosarcoma. Biochem Biophys Res Commun. 2017;49:217-24.

115. Chen J, Yu Y, Li H, Hu Q, Chen X, He Y, et al. Long non-coding RNA PVT1 promotes tumor progression by regulating the miR-143/HK2 axis in gallbladder cancer. Mol Cancer. 2019;18:1-16.

116. Zhu X, Du J, Gu Z. Circ-PVT1/miR-106a-5p/HK2 axis regulates cell growth, metastasis and glycolytic metabolism of oral squamous cell carcinoma. Mol Cell Biochem. 2020;474:147-58.

117. Yang YR, Zang SZ, Zhong CL, Li YX, Zhao SS, Feng XJ. Increased expression of the IncRNA PVT1 promotes tumorigenesis in non-small cell lung cancer. Int J Clin Exp Pathol. 2014;7:6929-35. 
118. Huang C, Liu S, Wang H, Zhang Z, Yang Q, Gao F. LncRNA PVT1 overexpression is a poor prognostic biomarker and regulates migration and invasion in small cell lung cancer. Am J Transl Res. 2016;8:5025-34.

\section{AUTHOR CONTRIBUTIONS}

DTr, GS and LSK designed the concept of the paper. DTr and GS performed literature research and wrote the first draft of the paper. CTS and DTo supervised the work. DTo, DTr and CTS design the figures. CTS, DTo, LSK, MG, GV, GMac and GMar critically revised the paper. All authors read and approved the final paper.

\section{FUNDING INFORMATION}

None.

\section{ETHICS APPROVAL AND CONSENT TO PARTICIPATE} Not applicable.

\section{CONSENT TO PUBLISH}

Not applicable.

\section{COMPETING INTERESTS}

The authors declare no competing interests.

\section{ADDITIONAL INFORMATION}

Supplementary information The online version contains supplementary material available at https://doi.org/10.1038/s41416-021-01584-7.

Correspondence and requests for materials should be addressed to Clelia Tiziana Storlazzi.

Reprints and permission information is available at http://www.nature.com/ reprints

Publisher's note Springer Nature remains neutral with regard to jurisdictional claims in published maps and institutional affiliations.

c) (i) Open Access This article is licensed under a Creative Commons Attribution 4.0 International License, which permits use, sharing, adaptation, distribution and reproduction in any medium or format, as long as you give appropriate credit to the original author(s) and the source, provide a link to the Creative Commons license, and indicate if changes were made. The images or other third party material in this article are included in the article's Creative Commons license, unless indicated otherwise in a credit line to the material. If material is not included in the article's Creative Commons license and your intended use is not permitted by statutory regulation or exceeds the permitted use, you will need to obtain permission directly from the copyright holder. To view a copy of this license, visit http://creativecommons. org/licenses/by/4.0/.

(c) The Author(s) 2021 